\title{
LAS REPRESENTACIONES SOCIALES DE LA RESPONSABILIDAD DE ESTUDIANTES UNIVERSITARIOS GUATEMALTECOS
}

\author{
Lic. Humberto Emilio Aguilera Arévalo ${ }^{1}$
}

\begin{abstract}
RESUMEN
La responsabilidad se considera un concepto clave en el siglo $\mathrm{XX}$, ya que establece una conexión entre el individuo y la sociedad. Estudios recientes analizan la responsabilidad como un concepto relacional que conecta al individuo con un suceso y con sus resultados. En ese sentido, la responsabilidad puede estar vinculada al punto de vista de Markova sobre la dialogicidad y las representaciones sociales, ya que activa la dinámica Ego-Alter-Objeto: ser responsable de algo (Ego), hacia alguien (Alter), y en relación con una instancia ( Objeto). Este trabajo analiza las representaciones sociales de la responsabilidad en una muestra de 296 estudiantes universitarios de Guatemala, a quienes se les aplicó las redes semánticas naturales con las palabras estímulo Yo responsable, Yo irresponsable, Guatemalteco responsable, Guatemalteco irresponsable, Europeo responsable y Europeo Irresponsable. Posteriormente se construyeron dos cuestionarios, uno sobre responsabilidad y el otro irresponsabilidad a nivel personal, endogrupal y exogrupal, en base a las redes semánticas obtenidas. Finalmente se realizaron análisis factoriales y análisis de cluster jerárquicos correspondientes.
\end{abstract}

Palabras clave: responsabilidad, representaciones sociales, guatemalteco

\begin{abstract}
The responsibility is a key concept in the twentieth century because it establishes a connection between the individual and society. Recent studies analyze the responsibility as a relational concept that connects the individual to an event and its outcomes. In that sense, the responsibility may be linked to Markova perspective on dialogicality and social representations because it activates the Ego-Alter-Object dynamic: being responsible for something (Ego) toward someone (Alter), and in relation to an instance (object). This paper analyzes the social representations of responsibility in a sample of 296 university students from Guatemala, who answered six stimulus words I Responsible, I Irresponsible, Responsible Guatemalan, Irresponsible Guatemalan, Responsible European and Irresponsible European, using the natural semantic networks technique. Subsequently two questionnaires were made; one about responsibility and irresponsibility on a personal, in group and out group level, based on semantic networks obtained. Finally, factor analysis and hierarchical cluster analysis were made.
\end{abstract}

Key words: responsibility, social representations, guatemalan

\footnotetext{
${ }^{1}$ Universidad Masaryk Brno, República Checa. Correo electrónico: aguilera99@ yahoo.com
} 


\section{INTRODUCCIÓN}

La responsabilidad es considerado un constructo fundamental de las interacciones humanas, debido a su naturaleza básica de "ser capaz de responder", estableciendo una conexión entre el individuo y la sociedad. Esto de hecho implica un contexto relacional entre lo que alguien demanda y la respuesta que el otro da. De esta manera, el concepto de responsabilidad adquiere un papel central en la relación entre las personas. El concepto de responsabilidad implica al menos tres relaciones: ser responsable de algo, hacia alguien, y en relación con una instancia (Bierhoff y Auhagen, 2001). En ese sentido, el término no se refiere a la responsabilidad que las personas tienen de sus propias acciones en actos de comisión, omisión y de las consecuencias de sus acciones, sino que es un fenómeno más amplio que está presente en todos los ámbitos de nuestras vidas: somos responsables de nuestra familia, de nuestros amigos, de nuestros hijos, nuestro trabajo, el estado y la sociedad.

El término responsabilidad, como McKeon (1957) señaló, empezó a aparecer en Europa en el siglo XVII y XVIII y su análisis llevó inevitablemente a las discusiones sobre el libre albedrío versus determinismo. De hecho, fue durante la Ilustración que se formó la idea del hombre como ser independiente de los fenómenos sobrenaturales y, al mismo tiempo profundamente arraigado de la historia y de la naturaleza. La responsabilidad en este sentido trata acerca de vivir interrelacionado con el contexto histórico y cultural (Schulz, 1988).

En el pasado la relación entre individuos y grupos se basó en el concepto del deber, enfocado en la idea de control y represión de la libertad individual, sin embargo en el siglo XX esto es asimilado por el concepto de la responsabilidad.

Tomando un enfoque global del fenómeno, diferentes culturas miran a la responsabilidad de diferentes maneras en cómo ser responsable. Winston (2001) señaló que el concepto occidental moderno de la responsabilidad nunca ha estado muy alejado de su raíz etimológica, que es la capacidad de responder. Si se va a otras culturas, hay diferentes nociones de responsabilidad, cada uno con su propia historia. Cada lengua, inevitablemente, sella el término responsabilidad con su propio ingenio y limitaciones. Para los antiguos griegos y japoneses de hoy, la palabra responsabilidad tiene fuertes connotaciones de culpabilidad que para la palabra en español o en inglés. Para los antiguos griegos una persona es responsable (aitios) cuando la culpa (aitia) por algo pesa sobre sus hombros. Por lo tanto Aristóteles no trata la responsabilidad como una virtud. Por otro lado, los japoneses modernos no suelen asumir la responsabilidad (sekinin) para el éxito, sino sólo para el fracaso. En su cultura, piensan acerca de la responsabilidad en situaciones en las que puede considerárseles responsables, es decir, en situaciones que les gustarían evitar. Sin embargo, las personas con un sentido de responsabilidad (sekininkan ga aru hito) son muy apreciadas, aunque sólo sea porque están seguros de hacer frente a situaciones embarazosas de una manera honesta y directa. Villegas-Reimers (1988) encontró sinónimos de responsabilidad, como la causalidad, la culpa, el juicio moral, el control, la disciplina personal en adolescentes venezolanos en una investigación que hizo desde la psicología social. En la transición política también existen controversias acerca de la noción de responsabilidad en ciertas culturas. Un psicólogo ruso, Andrei Brushlinski, (1994) investigó sobre la controversia en Rusia durante la transición a la democracia después de la caída del comunismo. El notó una tendencia general de que las personas no se sentían responsables por sus 
propias acciones. Pero en este caso la tendencia a no sentirse responsable, no se debió al énfasis en los derechos individuales. Era algo que se había prorrogado del pasado régimen comunista. Los psicólogos a menudo comentan que el sistema soviético totalitario, que controlaba tanto la vida individual como colectiva, daba a los individuos tan pocas responsabilidades que habían interiorizado una actitud de desesperanza aprendida. Analizando este problema, Brushlinski sostuvo que la década de 1990 fue el momento en que la gente rusa reabrió el debate sobre la moralidad y la responsabilidad haciendo un llamado a la restauración de las conductas sociales basadas en la responsabilidad.

En los estudios transculturales se ha documentado diversas formas en que los individuos conceptualizan el yo. Estos descubrimientos tienen implicaciones importantes para la comprensión de la atribución de responsabilidad y que también plantean importantes desafíos a la teoría. Los psicólogos culturales han encontrado que los individuos de los países occidentales tienden a ver el yo como un ente independiente, mientras que las personas en los países orientales consideran que el yo está estrechamente conectado a los individuos y al contexto en el que viven (Markus y Kitayama, 1991 ; Nisbett, 1998; Triandis, 1989). Shweder y Bourne (1982) les pidieron a estadounidenses indígenas y no indígenas que describieran a sus compañeros. Los investigadores encontraron que los estadounidenses no indígenas utilizan mayoritariamente rasgos atribucionales sin relacionar a sus compañeros a su contexto cuando los describieron, mientras que la mayoría de los estadounidenses indígenas utilizaron descripciones relacionadas al contexto como "él es egoísta solo cuando está en casa".

El constructo individualismo-colectivismo describe un síndrome cultural en el que los individualistas tienden a dar prioridad a las metas individuales y colectivistas ponen más énfasis en las metas del grupo y a las normas (Triandis, 1995a, 1995b). Este amplio e influyente constructo puede ser útil para entender las diferencias entre Guatemala y otros países. Bartos (2010) estudió la población de la República Checa y descubrió que se encuentra psicosocialmente orientada a lo colectivista. Para los checos es más característico estar orientado al igualitarismo que con respeto a la desigualdad. En la investigación mostró que personas mayores y personas con bajos estándares de vida están mayoritariamente orientadas a lo colectivista. Por el contrario, las personas más jóvenes, personas con educación superior y las personas económicamente estables están orientadas a lo individualista. Hofstede (2001) encontró que la población guatemalteca es altamente orientada a lo colectivista. El individualismo puntúa extremadamente muy bajo, lo que significa una sociedad de naturaleza colectivista y con sólidas relaciones en las que todo el mundo asume la responsabilidad de los demás miembros de su grupo.

La responsabilidad tiene múltiples y diferentes facetas. Uno de los estudios más importantes sobre el significado de la responsabilidad fue el de Hart (1968), en el que señalaba que el término tanto utilizado en el derecho penal como en ética y en el sentido común tenía cuatro tipos fundamentales: la responsabilidad de rol, la responsabilidad causal, la responsabilidad de agravio y la responsabilidad capacidad. El primero se refiere a la responsabilidad que se asume al asumir un rol determinado, asumiendo los deberes específicos para el rol determinado. El segundo se refiere a la atribución de responsabilidad como causa de algo, el tercero se trata de ser responsable de las propias 
acciones, mientras que el cuarto se centra en la idea de la capacidad, es decir, que una persona es responsable en la medida que es capaz de hacer algo.

El estudio de la responsabilidad en la psicología social fue influenciado principalmente por la teoría de la atribución (Heider, 1958; Jones y Davis, 1965; Kelley, 1967) y se focaliza en cómo la gente hace explicaciones causales: que tipo de información la gente utiliza para hacer inferencias causales, y lo que hacen con esta información para responder a las preguntas causales. Watson (1982) menciona que el sesgo de atribución fundamental ocurre cuando existe la tendencia a atribuir el comportamiento propio a causas situacionales y el los demás a causas disposicionales. Sin embargo, la conceptualización del constructo de la responsabilidad en términos de atribución causal ha sido insuficiente para entenderla en todos sus diferentes significados, por el enfoque cognitivo e individual que se ha utilizado.

Por otra parte, el término responsabilidad fue utilizado por lo general sin tener una definición muy clara del mismo. Fincham y Jaspars (1980) observaron que clarificar el constructo era necesario si se quería comprender a la ciencia y al mundo ordinario. De hecho, el análisis del concepto entró en una controversia teórica donde la palabra era confundida a menudo con ideas de imputabilidad y de responsabilidad de rendir cuentas. Estas dos palabras se refieren a diferentes aspectos de la responsabilidad: la imputabilidad se centra en el reconocimiento de las causas que producen un evento, la responsabilidad de rendir cuentas implica que la gente tiene que responder de sus propias acciones de acuerdo a las reglas y normas reconocidas en un contexto cultural y social específico (Zamperini, 1998).

En su estudio sobre la responsabilidad de rol, Hamilton (1978) sostiene que tomando en cuenta la responsabilidad de rol le proporciona al individuo algo como su "vestimenta social", a menudo olvidado por los teóricos de atribución. El autor de la investigación se centró en los roles sociales del actor, entendidos como los contextos normativos que atan al individuo con determinadas sanciones. En la perspectiva de Hamilton sobre los juicios de responsabilidad menciona que dependen tanto de lo que el actor hizo y de lo que el actor tenía que hacer dado su rol social. Con Hamilton (1986), la causalidad personal se convierte en la causalidad interpersonal y la responsabilidad se define principalmente en sus aspectos relacionales.

Un interesante campo de investigación, estudiando el concepto de responsabilidad en el campo de la atribución causal, tenía un enfoque peculiar sobre las explicaciones de sentido común dados por el estudio de "rendir cuentas" (Scott y Lyman, 1968), es decir las justificaciones y excusas que la gente utiliza en su vida cotidiana. Esta técnica mostró que la responsabilidad subyace en las explicaciones del sentido común, una cuestión que es de considerable interés en una perspectiva más amplia conectando la responsabilidad con la noción de las representaciones sociales, consideradas como teorías del sentido común (Moscovici, 1984).

El vínculo entre las representaciones y explicaciones fue hecho hace mucho tiempo por Fauconnet (1920), que entendía que la responsabilidad era parte del sistema de las representaciones colectivas, y lo cual fue recientemente reconsiderado por Moscovici (1981). Este autor argumenta que las representaciones sociales determinan cuando la gente busca explicaciones y por qué las personas son desafiadas a encontrar 
explicaciones, cuando los acontecimientos son incompatibles con sus representaciones (Hewstone, 1989).

En ese sentido, la responsabilidad puede ser considerada como un aspecto dinámico de la relación entre las personas y la realidad, poniendo en marcha y apoyando la búsqueda de explicaciones.

Este aspecto inherente dinámico y relacional del concepto de la responsabilidad es tomado en cuenta en el modelo triangular de la responsabilidad desarrollado por Schlenker, Britt, Pennington, Murphy y Doherty (1994). Este modelo estudia a la responsabilidad como un adherente que conecta a un individuo con un evento y con resultados. Esto condujo hacia un modelo de responsabilidad que incorpora las definiciones anteriores de este concepto en un marco coherente para la comprensión de las causas y los efectos de la responsabilidad. Según este modelo, la responsabilidad en un hecho determinado es una transacción entre un hecho concreto que se haya producido o anticipado, las prescripciones o normas que rigen el evento, y las imágenes de identidad individual relevantes al evento y a la normativa. De esta manera, la responsabilidad que un individuo siente está en función de la fuerza de los vínculos entre estos tres elementos y de la importancia atribuida por el individuo a los mismos.

De esta manera, el modelo triangular de la responsabilidad puede estar relacionado con la perspectiva de Ivana Markova sobre la dialogicidad y las representaciones sociales (Markova, 2003).

Markova afirma que una representación social es una forma del conocimiento del sentido común, que se construye acerca de los objetos sociales que generan tensiones y que llegan a ser relevantes en un contexto histórico y cultural determinado. De hecho, con sus palabras, "las representaciones sociales son construidas sobre esos fenómenos sociales, objeto de preocupación pública, de los que la gente piensa, discute, que causan tensiones y favorecen a la acción" (Markova, 2003, 143). La noción de tensión adquiere una nueva relevancia en la perspectiva Ego-Alter-Objeto: lo que hace dinámico el triángulo semiótico de Moscovici, es la presencia de tensión. Con la tensión se tiene una tríada dialógica, "la unidad dinámica de la teoría del conocimiento social"(Markova, 2003, 153.). Hoy en día, la responsabilidad es un objeto de interés público siempre presente en la vida cotidiana de la gente y se adhiere plenamente a la perspectiva dialógica, ya que activa la dinámica Ego-Alter-Objeto: ser responsable de algo (Ego), hacia alguien (Alter), y en relación a una instancia (Objeto).

Por todas estas razones, se piensa en la noción de responsabilidad como un objeto de representación social y por ello se ha explorado el conocimiento del sentido común en el área semántica.

El objetivo de esta investigación es el análisis de las representaciones sociales de la responsabilidad en una muestra de estudiantes universitarios. 


\section{METODOLOGÍA}

\section{Muestra}

Se trabajó con una muestra no probabilística de cuota compuesta por 2 grupos de 148 sujetos de ambos sexos, de las carreras de ingenierías y humanidades de 2 universidades privadas de Guatemala.

La muestra estuvo compuesta de 296 estudiantes universitarios (Edad Media $=$ 21,7, Desviación Estándar = 5,07), de los cuales el 190 eran mujeres (el 64\%, Edad M = 22,14, Desviación Estándar = 5,95) y 106 eran hombres (el 36\%, Edad M = 20,91, Desviación Estándar = 2,78)

El 94,3\% de los estudiantes eran solteros y el 96,6\% originarios de la ciudad capital. El $80.5 \%$ eran exclusivamente estudiantes, es decir que no trabajaban, y el $81,7 \%$ vivían con sus padres. El 81,5\% de sus padres eran casados. El 72,4\% de sus padres eran universitarios, sin embargo un $22,9 \%$ habían estudiado bachillerato. Un $50,5 \%$ de sus madres eran universitarios, sin embargo un 39,9\% habían estudiado bachillerato.

Un $12.4 \%$ de los padres de los sujetos eran gerentes y un $12 \%$ eran ingenieros. Los estudiantes de ingeniería tenían más padres gerentes que los de humanidades por un $4.4 \%$

Un $37.3 \%$ de las madres de los sujetos eran amas de casa y un $6.7 \%$ eran maestras. Los estudiantes de humanidades tenían más madres amas de casa que los de humanidades por un $2.8 \%$

El 70.6\% de los sujetos conocían a un europeo. De ese $70.6 \%$ de los que conocían a un europeo, un $23.4 \%$ conocían a un español, un $15.9 \%$ a un alemán y un $14.1 \%$ a un francés

Los estudiantes de ingeniería conocían por un $2.2 \%$ más a españoles y por un $2.8 \%$ más a un francés.

\section{Instrumentos}

Se aplicaron 3 cuestionarios que comprenden:

RedessemánticasnaturalesdeValdezMedina: Para las palabras estímulo Yo responsable, Yo irresponsable, Guatemalteco responsable, Guatemalteco irresponsable, Europeo responsable, Europeo irresponsable

Cuestionario deresponsabilidad: Se diseñó un cuestionario tipo Likert, de 50 reactivos con cinco opciones de respuesta (muy fuerte, fuerte, media, débil y no tiene relación). Del total de reactivos 25 fueron tomados de las redes semánticas naturales obtenidas para Yo responsable y 25 más obtenidas de Yo irresponsable. El criterio de selección de los 50 reactivos fue que las palabras definidoras elegidas tuvieran el mayor peso 
semántico o valor M de cada grupo respectivamente. De la misma forma se hizo un cuestionario para Yo irresponsable, Guatemalteco responsable e irresponsable y Europeo responsable e irresponsable.

Cuestionariodeautoconceptoderesponsabilidad: Se diseñó un cuestionario tipo Likert, de 50 reactivos con cinco opciones de respuesta (lo tengo totalmente, tengo algo de esta característica, ni lo tengo ni me falta, tengo poco de esta característica y no tengo nada de esta característica). Del total de reactivos 25 fueron tomados de las redes semánticas naturales obtenidas para Yo responsable y 25 más obtenidas de Yo irresponsable. El criterio de selección de los 50 reactivos fue que las palabras definidoras elegidas tuvieran el mayor peso semántico o valor $\mathrm{M}$ de cada grupo respectivamente. De la misma forma se hizo un cuestionario de autoconcepto de responsabilidad del Guatemalteco y un último del Europeo.

\section{Procedimiento}

En la aplicación de las redes semánticas naturales se les pidió a los sujetos que definieran con un mínimo de cinco palabras sueltas que podían ser: verbos, adverbios, sustantivos, adjetivos, etc., sin usar partículas gramaticales como preposiciones o artículos, a cada una de las palabras estímulo (Yo responsable, Yo irresponsable, Guatemalteco responsable, Guatemalteco irresponsable, Europeo responsable, Europeo irresponsable)

Posteriormente, se les pidió que jerarquizaran cada una de las palabras que dieron como definidoras, asignándole el número 1 a la palabra que consideraran que estuviera más cerca, más relacionada o que mejor definiera a la palabra estímulo, el número 2 a la que le sigue en relación, el 3 a la siguiente y así sucesivamente hasta terminar de jerarquizar todas y cada una de las palabras definidoras generadas por los sujetos.

En la aplicación de los cuestionarios de responsabilidad se les pidió a los sujetos que indicaran el grado de relación de cada uno de los reactivos con la palabra estímulo Yo responsable. De la misma forma se hizo con los siguientes 5 cuestionarios de Yo irresponsable, Guatemalteco responsable, Guatemalteco irresponsable, Europeo responsable, Europeo irresponsable

En la aplicación de los cuestionarios de autoconcepto de responsabilidad se les pidió a los sujetos que indicaran que tanto tenían de cada uno de los reactivos del cuestionario. De la misma manera se hizo con el autoconcepto de responsabilidad del Guatemalteco y del Europeo.

Los cuestionarios fueron distribuidos en 2 universidades privadas ya que se asumió que los estudiantes habían tenido un mayor contacto con alguna persona europea que si se tratara de la universidad nacional. La participación fue voluntaria y la prueba se llenó de manera individual. Los datos fueron recolectados entre febrero y mayo del 2010. 


\section{Análisis}

Se realizó el análisis respectivo en las redes semánticas naturales, obteniendo el grupo SAM, es decir el grupo de 15 palabras definidoras con mayor peso semántico, de cada palabra estímulo. Posteriormente se obtuvo el grupo SAM respectivo de los estudiantes de ingenierías e humanidades.

Posteriormente se realizó un análisis de clusters jerárquicos de cada cuestionario, tomando como criterio el método de unión de máximas distancias, usando como medida el cuadrado de la distancia euclidiana.

Finalmente se realizó un análisis factorial exploratorio por cada palabra estímulo, así como también para los cuestionarios de Autoconcepto de responsabilidad personal, endogrupal y exogrupal, sometiendo los reactivos a rotación varimax, tomando como criterio que el valor Eigen o autovalor del factor fuera mayor a uno, y que los pesos factoriales de los reactivos fueran mayor a 0.3. A continuación, con los resultados obtenidos en el análisis factorial se utilizó la correlación de Spearman Brown, entre los valores $\mathrm{M}$ obtenidos para las palabras definidoras encontradas a través de las redes semánticas naturales y los pesos factoriales obtenidos para las mismas palabras (coincidentes), obtenidos a través del análisis factorial, con la finalidad de observar el grado de relación que había entre ellas, y con ello validar el uso de la técnica.

También se realizó el análisis de confiabilidad general y por factores. De ellos se calcularon su promedio, desviación estándar y sus correlaciones.

\section{Hipótesis}

Ho: Los estudiantes reconocen la multiplicidad de significados del término responsabilidad en la dinámica Ego (en el sentido del rol) - Alter ( en el sentido de la relación con otros) - Objeto (en el sentido de las obligaciones y tareas específicas).

H1: Los estudiantes no reconocen la multiplicidad de significados del término responsabilidad en la dinámica Ego (en el sentido del rol) - Alter ( en el sentido de la relación con otros) - Objeto (en el sentido de las obligaciones y tareas específicas). 


\section{RESULTADOS}

A partir de los datos obtenidos por las redes semánticas naturales se obtuvo que para la palabra estímulo Yo responsable, la riqueza semántica fue de 500 palabras y se encontró que fue definido por los sujetos como puntual, cumplido, ordenado, trabajador, organizado, honesto, dedicado, respetuoso, estudioso, trabajo, tareas, estudio, comprometido, atento y disciplina (ver tabla 1).

\begin{tabular}{|l|r|r|}
\hline \multicolumn{3}{|c|}{ Tabla 1 } \\
Conjunto SAM para YO RESPONSABLE \\
\hline \begin{tabular}{l|r|r|} 
CONJUNTO \\
SAM
\end{tabular} & $\begin{array}{l}\text { VALOR } \\
\text { M }\end{array}$ & VALOR FMG \\
\hline puntual & 1088 & $100 \%$ \\
\hline cumplido & 715 & $66 \%$ \\
\hline ordenado & 581 & $53 \%$ \\
\hline trabajador & 562 & $52 \%$ \\
\hline organizado & 505 & $46 \%$ \\
\hline honesto & 504 & $46 \%$ \\
\hline dedicado & 471 & $43 \%$ \\
\hline respetuoso & 330 & $30 \%$ \\
\hline estudioso & 322 & $30 \%$ \\
\hline trabajo & 310 & $28 \%$ \\
\hline tareas & 291 & $27 \%$ \\
\hline estudio & 266 & $24 \%$ \\
\hline comprometido & 247 & $23 \%$ \\
\hline atento & 211 & $19 \%$ \\
\hline disciplina & 194 & $18 \%$ \\
\hline j=500 & & \\
\hline
\end{tabular}

En el conjunto SAM para Yo responsable por carrera se observó que ambos grupos consideraron puntual y cumplido como las palabras mayor definidoras, sin embargo los estudiantes de humanidades consideraron más importante organizado, trabajo, respetuoso, estudio, comprometido y atento que los de ingeniería. Los estudiantes de ingeniería consideraron más importantes trabajador, honesto, estudioso, disciplina y tareas que los estudiantes de humanidades (ver tabla 2). 


\begin{tabular}{|l|r|l|r|l|r|}
\hline \multicolumn{5}{|c|}{ Conjunto SAM para YO RESPONSAB } \\
\hline $\begin{array}{l}\text { HUMANIDADE } \\
\text { S }\end{array}$ & VMT & INGENIERÍAS & VMT & GENERAL & VMT \\
\hline puntual & 541 & puntual & 547 & puntual & 1088 \\
\hline cumplido & 348 & cumplido & 367 & cumplido & 715 \\
\hline organizado & 276 & trabajador & 346 & ordenado & 581 \\
\hline ordenado & 249 & ordenado & 332 & trabajador & 562 \\
\hline trabajador & 216 & honesto & 301 & organizado & 505 \\
\hline dedicado & 216 & dedicado & 255 & honesto & 504 \\
\hline honesto & 203 & estudioso & 230 & dedicado & 471 \\
\hline trabajo & 193 & organizado & 229 & respetuoso & 330 \\
\hline respetuoso & 191 & disciplina & 164 & estudioso & 322 \\
\hline estudio & 145 & tareas & 156 & trabajo & 310 \\
\hline tareas & 135 & respetuoso & 139 & tareas & 291 \\
\hline comprometido & 129 & estudio & 121 & estudio & 266 \\
\hline atento & 97 & comprometido & 118 & comprometido & 247 \\
\hline estudioso & 92 & trabajo & 117 & atento & 211 \\
\hline disciplina & 30 & atento & 114 & disciplina & 194 \\
\hline N=296 & & & & & \\
\hline
\end{tabular}

A partir de los datos obtenidos por las redes semánticas naturales se obtuvo que para la palabra estímulo Guatemalteco responsable, la riqueza semántica fue de 552 palabras y se encontró que fue definido por los sujetos como trabajador, puntual, cumplido, respetuoso, honesto, trabajo, ordenado, organizado, honrado, dedicado, consciente, patriota, solidario, amable y familia (ver tabla 3).

\begin{tabular}{|l|r|r|}
\hline \multicolumn{3}{|c|}{ Tabla 3 } \\
RESPONSABLE \\
Conjunto SAM para GUATEMALTECO \\
CONJUNTO SAM & $\begin{array}{l}\text { VALOR } \\
\text { M }\end{array}$ & VALOR FMG \\
\hline trabajador & 1047 & $100 \%$ \\
\hline puntual & 651 & $62 \%$ \\
\hline cumplido & 486 & $46 \%$ \\
\hline respetuoso & 413 & $39 \%$ \\
\hline honesto & 395 & $38 \%$ \\
\hline trabajo & 338 & $32 \%$ \\
\hline ordenado & 322 & $31 \%$ \\
\hline organizado & 294 & $28 \%$ \\
\hline honrado & 203 & $19 \%$ \\
\hline dedicado & 187 & $18 \%$ \\
\hline consciente & 186 & $18 \%$ \\
\hline patriota & 175 & $17 \%$ \\
\hline solidario & 172 & $16 \%$ \\
\hline amable & 158 & $15 \%$ \\
\hline familia & 151 & $14 \%$ \\
\hline j = 552 & \multicolumn{2}{|c|}{} \\
\cline { 1 - 2 } &
\end{tabular}

En el conjunto SAM para Guatemalteco responsable por carrera se observó que ambos grupos consideraron trabajador, puntual y cumplido como las palabras mayor definidoras, sin embargo los estudiantes de humanidades consideraron más importante organizado, trabajo, honrado, patriota, amable, familia que los de ingeniería. Los 
estudiantes de ingeniería consideraron más importantes respetuoso, honesto, ordenado, consciente, dedicado y solidario que los estudiantes de humanidades (ver tabla 4).

\begin{tabular}{|l|r|l|r|l|r|}
\hline \multicolumn{7}{|c|}{ Tabla 4 } \\
\hline HUMANIDADES & VMT & INGENIERÍAS & VMT & GENERAL & VMT \\
\hline trabajador & 503 & trabajador & 544 & trabajador & 1047 \\
\hline puntual & 319 & puntual & 332 & puntual & 651 \\
\hline cumplido & 230 & cumplido & 256 & cumplido & 486 \\
\hline organizado & 198 & respetuoso & 253 & respetuoso & 413 \\
\hline trabajo & 179 & honesto & 239 & honesto & 395 \\
\hline respetuoso & 160 & ordenado & 191 & trabajo & 338 \\
\hline honesto & 156 & trabajo & 159 & ordenado & 322 \\
\hline honrado & 152 & consciente & 101 & organizado & 294 \\
\hline ordenado & 131 & organizado & 96 & honrado & 203 \\
\hline patriota & 101 & dedicado & 93 & dedicado & 187 \\
\hline dedicado & 94 & solidario & 88 & consciente & 186 \\
\hline amable & 91 & patriota & 74 & patriota & 175 \\
\hline familia & 88 & amable & 67 & solidario & 172 \\
\hline consciente & 85 & familia & 63 & amable & 158 \\
\hline solidario & 84 & honrado & 51 & familia & 151 \\
\hline n=296 & & & & & \\
\hline
\end{tabular}

A partir de los datos obtenidos por las redes semánticas naturales se obtuvo que para la palabra estímulo Europeo responsable, la riqueza semántica fue de 503 palabras y se encontró que fue definido por los sujetos como puntual, trabajador, respetuoso, organizado, trabajo, cumplido, educado, ordenado, honesto, limpio, consciente, estudioso, exitoso, amable, cultura (ver tabla 5).

\begin{tabular}{|l|r|r|}
\hline \multicolumn{3}{|c|}{$\begin{array}{c}\text { Cobla 5 } \\
\text { Conto SAM para EUROPEO } \\
\text { RESPONSABLE }\end{array}$} \\
\hline $\begin{array}{l}\text { CONJUNTO } \\
\text { SAM }\end{array}$ & $\begin{array}{l}\text { VALOR } \\
\text { M }\end{array}$ & VALOR FMG \\
\hline puntual & 755 & $100 \%$ \\
\hline trabajador & 650 & $86 \%$ \\
\hline respetuoso & 338 & $45 \%$ \\
\hline organizado & 281 & $37 \%$ \\
\hline trabajo & 277 & $37 \%$ \\
\hline cumplido & 249 & $33 \%$ \\
\hline educado & 245 & $32 \%$ \\
\hline ordenado & 241 & $32 \%$ \\
\hline honesto & 238 & $32 \%$ \\
\hline limpio & 170 & $23 \%$ \\
\hline consciente & 150 & $20 \%$ \\
\hline estudioso & 138 & $18 \%$ \\
\hline exitoso & 121 & $16 \%$ \\
\hline amable & 121 & $16 \%$ \\
\hline cultura & 120 & $16 \%$ \\
\hline j=503 & & \\
\hline
\end{tabular}

En el conjunto SAM para Europeo responsable por carrera se observó que ambos grupos consideraron puntual y trabajador como las palabras mayor definidoras, sin embargo los estudiantes de humanidades consideraron más importante organizado, 
educado, ordenado, amable, estudioso que los de ingeniería. Los estudiantes de ingeniería consideraron más importantes respetuoso, trabajo, honesto, cumplido, limpio, consciente, exitoso y cultura que los estudiantes de humanidades (ver tabla 6).

\begin{tabular}{|c|c|c|c|c|c|}
\hline \multicolumn{6}{|c|}{$\begin{array}{c}\text { Tabla } 6 \\
\text { Conjunto SAM para EUROPEO RESPONSABLE por carrera }\end{array}$} \\
\hline HUMANIDADES & VMT & INGENIERIIAS & $\begin{array}{l}M M \\
T \\
\end{array}$ & GENERAL & VMT \\
\hline puntual & 317 & puntual & 438 & puntual & 755 \\
\hline trabajador & 311 & trabajador & 339 & trabajador & 650 \\
\hline organizado & 153 & respetuoso & 201 & respetuoso & 338 \\
\hline educado & 152 & trabajo & 161 & organizado & 281 \\
\hline respetuoso & 137 & honesto & 155 & trabajo & 277 \\
\hline ordenado & 132 & cumplido & 135 & cumplido & 249 \\
\hline trabajo & 116 & organizado & 128 & educado & 245 \\
\hline cumplido & 114 & limpio & 116 & ordenado & 241 \\
\hline honesto & 83 & ordenado & 109 & honesto & 238 \\
\hline amable & 68 & consciente & 104 & limpio & 170 \\
\hline estudioso & 62 & educado & 93 & consciente & 150 \\
\hline limpio & 54 & exitoso & 93 & estudioso & 138 \\
\hline consciente & 46 & cultura & 93 & exitoso & 121 \\
\hline exitoso & 28 & estudioso & 76 & amable & 121 \\
\hline cultura & 27 & amable & 53 & cultura & 120 \\
\hline$n=296$ & & & & & \\
\hline
\end{tabular}

A partir de los datos obtenidos por las redes semánticas naturales se obtuvo que para la palabra estímulo Yo irresponsable, la riqueza semántica fue de 591 palabras y se encontró que fue definido por los sujetos como impuntual, desordenado, haragán, desorganizado, incumplido, perezoso, dejado, olvidadizo, irrespetuoso, descuidado, despreocupado, desinteresado, deshonesto, mentiroso y malo (ver tabla 7).

\begin{tabular}{|l|r|r|}
\hline \multicolumn{3}{|c|}{ Tabla 7 } \\
\hline Conjunto SAM para YO IRRESPONSABLE \\
\hline $\begin{array}{l}\text { CONJUNTO } \\
\text { SAM }\end{array}$ & \multicolumn{1}{|l|}{ VALOR } & M \\
\hline impuntual & 887 & $100 \%$ \\
\hline desordenado & 732 & $83 \%$ \\
\hline haragán & 597 & $67 \%$ \\
\hline desorganizado & 511 & $58 \%$ \\
\hline incumplido & 455 & $51 \%$ \\
\hline perezoso & 361 & $41 \%$ \\
\hline dejado & 273 & $31 \%$ \\
\hline olvidadizo & 265 & $30 \%$ \\
\hline irrespetuoso & 241 & $27 \%$ \\
\hline descuidado & 234 & $26 \%$ \\
\hline despreocupado & 228 & $26 \%$ \\
\hline desinteresado & 218 & $25 \%$ \\
\hline deshonesto & 188 & $21 \%$ \\
\hline mentiroso & 143 & $16 \%$ \\
\hline malo & 133 & $15 \%$ \\
\hline j=591 & \multicolumn{2}{|l}{} \\
\hline
\end{tabular}


En el conjunto SAM para Yo irresponsable por carrera se observó que ambos grupos consideraron impuntual y desordenado como las palabras mayor definidoras, sin embargo los estudiantes de humanidades consideraron más importantes desorganizado, irrespetuoso, descuidado, despreocupado y malo que los de ingeniería. Los estudiantes de ingeniería consideraron más importantes haragán, incumplido, dejado, desinteresado y mentiroso que los estudiantes de humanidades (ver tabla 8).

\begin{tabular}{|c|c|c|c|c|c|}
\hline \multicolumn{6}{|c|}{$\begin{array}{c}\text { Tabla } 8 \\
\text { Conjunto SAM para YO IRRESPONSABLE por carrera }\end{array}$} \\
\hline HUMANIDADES & $V M T$ & INGENIERÍAS & VMT & GENERAL & VMT \\
\hline impuntual & 431 & impuntual & 456 & impuntual & 887 \\
\hline desordenado & 357 & desordenado & 375 & desordenado & 732 \\
\hline desorganizado & 297 & haragán & 356 & haragán & 597 \\
\hline Haragán & 241 & incumplido & 246 & desorganizado & 511 \\
\hline incumplido & 209 & desorganizado & 214 & incumplido & 455 \\
\hline perezoso & 171 & perezoso & 190 & perezoso & 361 \\
\hline irrespetuoso & 129 & dejado & 150 & dejado & 273 \\
\hline olvidadizo & 128 & olvidadizo & 137 & olvidadizo & 265 \\
\hline Dejado & 123 & desinteresado & 118 & irrespetuoso & 241 \\
\hline descuidado & 122 & irrespetuoso & 112 & descuidado & 234 \\
\hline despreocupado & 119 & descuidado & 112 & $\begin{array}{l}\text { despreocupad } \\
0\end{array}$ & 228 \\
\hline desinteresado & 100 & despreocupado & 109 & desinteresado & 218 \\
\hline deshonesto & 82 & deshonesto & 106 & deshonesto & 188 \\
\hline Malo & 78 & mentiroso & 78 & mentiroso & 143 \\
\hline mentiroso & 65 & malo & 55 & malo & 133 \\
\hline$n=296$ & & & & & \\
\hline
\end{tabular}

A partir de los datos obtenidos por las redes semánticas naturales se obtuvo que para la palabra estímulo Guatemalteco irresponsable, la riqueza semántica fue de 576 palabras y se encontró que fue definido por los sujetos como impuntual, haragán, desordenado, irrespetuoso, incumplido, perezoso, desorganizado, mentiroso, deshonesto, desinteresado, ladrón, corrupto, dejado, despreocupado, vago(ver tabla 9). 


\begin{tabular}{|l|r|r|}
\hline \multicolumn{3}{|c|}{$\begin{array}{l}\text { Tabla 9 } \\
\text { Conjunto SAM para GUATEMALTECO } \\
\text { IRRESPONSABLE }\end{array}$} \\
\hline $\begin{array}{l}\text { CONJUNTO } \\
\text { SAM }\end{array}$ & $\begin{array}{l}\text { VALOR } \\
\text { M }\end{array}$ & VALOR FMG \\
\hline impuntual & 934 & $100 \%$ \\
\hline haragán & 693 & $74 \%$ \\
\hline desordenado & 282 & $30 \%$ \\
\hline irrespetuoso & 279 & $30 \%$ \\
\hline incumplido & 274 & $29 \%$ \\
\hline perezoso & 269 & $29 \%$ \\
\hline desorganizado & 260 & $28 \%$ \\
\hline mentiroso & 243 & $26 \%$ \\
\hline deshonesto & 235 & $25 \%$ \\
\hline desinteresado & 228 & $24 \%$ \\
\hline ladrón & 223 & $24 \%$ \\
\hline corrupto & 220 & $24 \%$ \\
\hline dejado & 214 & $23 \%$ \\
\hline despreocupado & 205 & $22 \%$ \\
\hline vago & 196 & $21 \%$ \\
\hline j=576 & & \\
\hline
\end{tabular}

En el conjunto SAM para Guatemalteco irresponsable por carrera se observó que ambos grupos consideraron impuntual y haragán como las palabras mayor definidoras, sin embargo los estudiantes de humanidades consideraron más importantes desordenado, dejado, irrespetuoso, desorganizado, deshonesto, desinteresado y vago que los de ingeniería. Los estudiantes de ingeniería consideraron más importantes mentiroso, corrupto, ladrón, incumplido, perezoso y despreocupado que los estudiantes de humanidades (ver tabla 10).

\begin{tabular}{|l|r|l|r|l|l|}
\hline \multicolumn{7}{|c|}{ Tabla 10 } \\
Conjunto SAM para GUATEMALTECO IRRESPONSABLE por carrera \\
\hline HUMANIDADES & VMT & INGENIERÍAS & VMT & GENERAL & VMT \\
\hline impuntual & 485 & impuntual & 449 & impuntual & 934 \\
\hline haragán & 266 & haragán & 427 & haragán & 693 \\
\hline desordenado & 166 & mentiroso & 159 & desordenado & 282 \\
\hline dejado & 142 & corrupto & 153 & irrespetuoso & 279 \\
\hline irrespetuoso & 138 & ladrón & 152 & incumplido & 274 \\
\hline desorganizado & 133 & incumplido & 143 & perezoso & 269 \\
\hline deshonesto & 133 & irrespetuoso & 141 & desorganizado & 260 \\
\hline incumplido & 131 & perezoso & 141 & mentiroso & 243 \\
\hline perezoso & 128 & desorganizado & 127 & deshonesto & 235 \\
\hline desinteresado & 118 & despreocupado & 126 & desinteresado & 228 \\
\hline Vago & 98 & desordenado & 116 & ladrón & 223 \\
\hline mentiroso & 84 & desinteresado & 110 & corrupto & 220 \\
\hline despreocupado & 79 & deshonesto & 102 & dejado & 214 \\
\hline ladrón & 71 & vago & 98 & despreocupado & 205 \\
\hline corrupto & 67 & dejado & 72 & vago & 196 \\
\hline n=296 & & & & & \\
\hline
\end{tabular}

A partir de los datos obtenidos por las redes semánticas naturales se obtuvo que para la palabra estímulo Europeo irresponsable, la riqueza semántica fue de 501 palabras y se encontró que fue definido por los sujetos como impuntual, haragán, 
drogas, irrespetuoso, desordenado, sucio, desorganizado, vago, incumplido, fiestero, egoísta, desinteresado, dejado, descuidado y perezoso (ver tabla 11).

\begin{tabular}{|l|r|r|}
\hline \multicolumn{3}{|c|}{$\begin{array}{c}\text { Conjunto } \\
\text { TRAM para EUROPEO } \\
\text { IRRESPONSABE }\end{array}$} \\
\hline $\begin{array}{l}\text { CONJUNTO } \\
\text { SAM }\end{array}$ & $\begin{array}{l}\text { VALOR } \\
\text { M }\end{array}$ & VALOR FMG \\
\hline impuntual & 364 & $100 \%$ \\
\hline haragán & 355 & $98 \%$ \\
\hline drogas & 231 & $63 \%$ \\
\hline irrespetuoso & 229 & $63 \%$ \\
\hline desordenado & 224 & $62 \%$ \\
\hline sucio & 210 & $58 \%$ \\
\hline desorganizado & 208 & $57 \%$ \\
\hline vago & 187 & $51 \%$ \\
\hline incumplido & 178 & $49 \%$ \\
\hline fiestero & 172 & $47 \%$ \\
\hline egoísta & 170 & $47 \%$ \\
\hline desinteresado & 166 & $46 \%$ \\
\hline dejado & 152 & $42 \%$ \\
\hline descuidado & 150 & $41 \%$ \\
\hline perezoso & 144 & $40 \%$ \\
\hline j=501 & & \\
\hline
\end{tabular}

En el conjunto SAM para Europeo irresponsable por carrera se observó que ambos grupos difirieron respeto a las palabras mayor definidoras, donde los estudiantes de humanidades consideraron más importantes impuntual, desordenado, desorganizado, irrespetuoso, desinteresado, incumplido, dejado y descuidado que los de ingeniería. Los estudiantes de ingeniería consideraron más importantes drogas, fiestero, sucio, vago, egoísta y perezoso que los estudiantes de humanidades (ver tabla 12).

\begin{tabular}{|l|r|l|l|l|l|}
\hline \multicolumn{5}{|c|}{ Tabla 12 } \\
\hline $\begin{array}{l}\text { Conjunto SAM para EUROPEO IRRESPONSABLE por carrera } \\
\text { S }\end{array}$ & VMT & INGENIERÍAS & $\begin{array}{l}\text { VM } \\
T\end{array}$ & GENERAL & $\begin{array}{l}\text { VM } \\
T\end{array}$ \\
\hline impuntual & 169 & haragán & 275 & impuntual & 364 \\
\hline desordenado & 123 & drogas & 196 & haragán & 355 \\
\hline desorganizado & 119 & impuntual & 195 & drogas & 231 \\
\hline irrespetuoso & 102 & fiestero & 144 & irrespetuoso & 229 \\
\hline desinteresado & 85 & sucio & 141 & desordenado & 224 \\
\hline incumplido & 82 & Vago & 128 & sucio & 210 \\
\hline haragán & 80 & irrespetuoso & 127 & desorganizado & 208 \\
\hline dejado & 80 & egoísta & 112 & vago & 187 \\
\hline sucio & 69 & desordenado & 101 & incumplido & 178 \\
\hline descuidado & 61 & perezoso & 97 & fiestero & 172 \\
\hline vago & 59 & incumplido & 96 & egoísta & 170 \\
\hline egoísta & 58 & desorganizado & 89 & desinteresado & 166 \\
\hline perezoso & 47 & descuidado & 89 & dejado & 152 \\
\hline drogas & 35 & desinteresado & 81 & descuidado & 150 \\
\hline fiestero & 28 & dejado & 72 & perezoso & 144 \\
\hline n=296 & & & & & \\
\hline
\end{tabular}


A partir de los resultados obtenidos en el análisis de cluster jerárquico para analizar la estructura común de los individuos de la palabra estímulo Yo responsable se encontraron 2 grandes grupos que están más alejados entre sí, donde el grupo A se conforma por las palabras comprometido, cumplido, organizado, consciente, disciplina, trabajador, esfuerzo, puntual, trabajo, dedicado, ordenado, aplicado, estudioso, cuidadoso, correcto, honesto, confiable, estudio, universidad y tareas. El grupo B se conforma por las palabras: atento, inteligente, limpio, respetuoso y familia. El grupo A puede subdividirse en 2 , donde queda conformado por el grupo A1 compuesto por: comprometido, cumplido, organizado, consciente, disciplina, trabajador, esfuerzo, puntual, trabajo, dedicado, ordenado, aplicado, estudioso, cuidadoso y por el grupo A2 donde queda compuesto por las palabras: correcto, honesto, confiable, estudio, universidad y tareas. El grupo B se puede dividir en B1 conformado por: atento, inteligente y B2 por: limpio, respetuoso y familia. Las palabras más cercanas entre sí del grupo A son cumplido-comprometido y aplicado-estudios, mientras que las palabras más cercanas entre sí del grupo B son limpio-respetuoso, atento-inteligente (ver tabla 13)

TABLA 13

Dendrograma de Yo responsable utilizando el método de unión de máximas distancias Rescaled Distance Cluster Combine

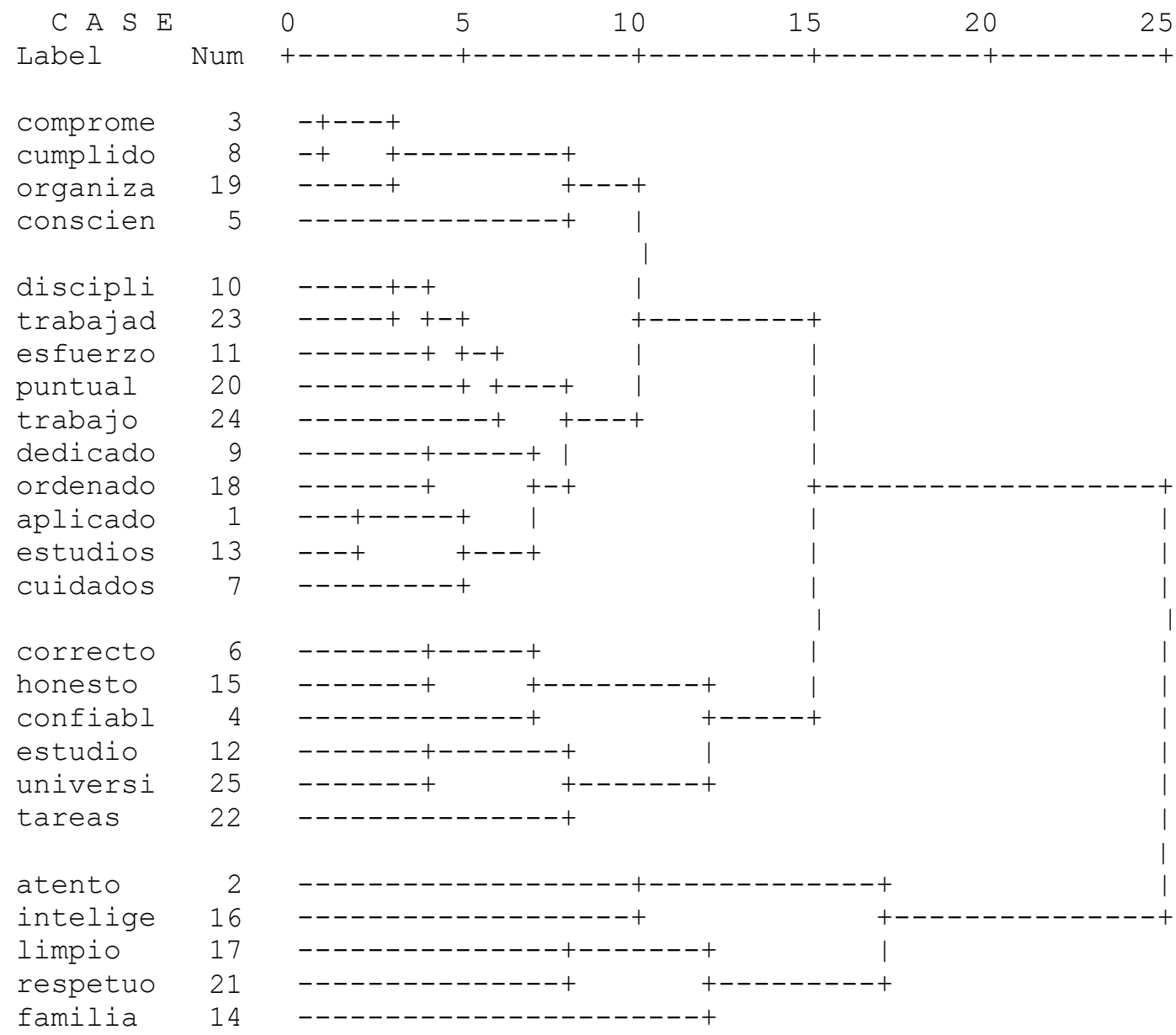


analizar la estructura común de los individuos de la palabra estímulo Guatemalteco responsable se encontraron 2 grandes grupos que están más alejados entre sí, donde el grupo A se conforma por las palabras: cumplido, honesto, dedicado, esfuerzo, trabajador, trabajo, consciente, luchador, atento, colaborador, familia, ordenado, organizado, estudios, puntual. El grupo B queda conformado por las palabras: amable, inteligente, educado, limpio, respetuoso, solidario, honrado, justo, ayudar y patriota. El grupo A se subdivide en 2 grupos, donde el grupo A1 queda conformado por: cumplido, honesto, dedicado, esfuerzo, trabajador, trabajo, consciente, luchador, atento, colaborador, familia. El grupo A2 queda conformado por: ordenado, organizado, estudios y puntual. El grupo B puede dividirse en B1 conformado por: amable, inteligente, educado y limpio, B2 conformado por: respetuoso, solidario, honrado, justo y ayudar y B3 conformado por: patriota. Las palabras más cercanas entre sí en el grupo A son: cumplido-honesto, dedicado-esfuerzo y para el grupo B: honrado-justo. Se puede observar que la palabra patriota es la palabra más alejada del resto de palabras del grupo B (ver tabla 14)

TABLA 14

Dendrograma de Guatemalteco responsable utilizando el método de unión de máximas distancias Rescaled Distance Cluster Combine

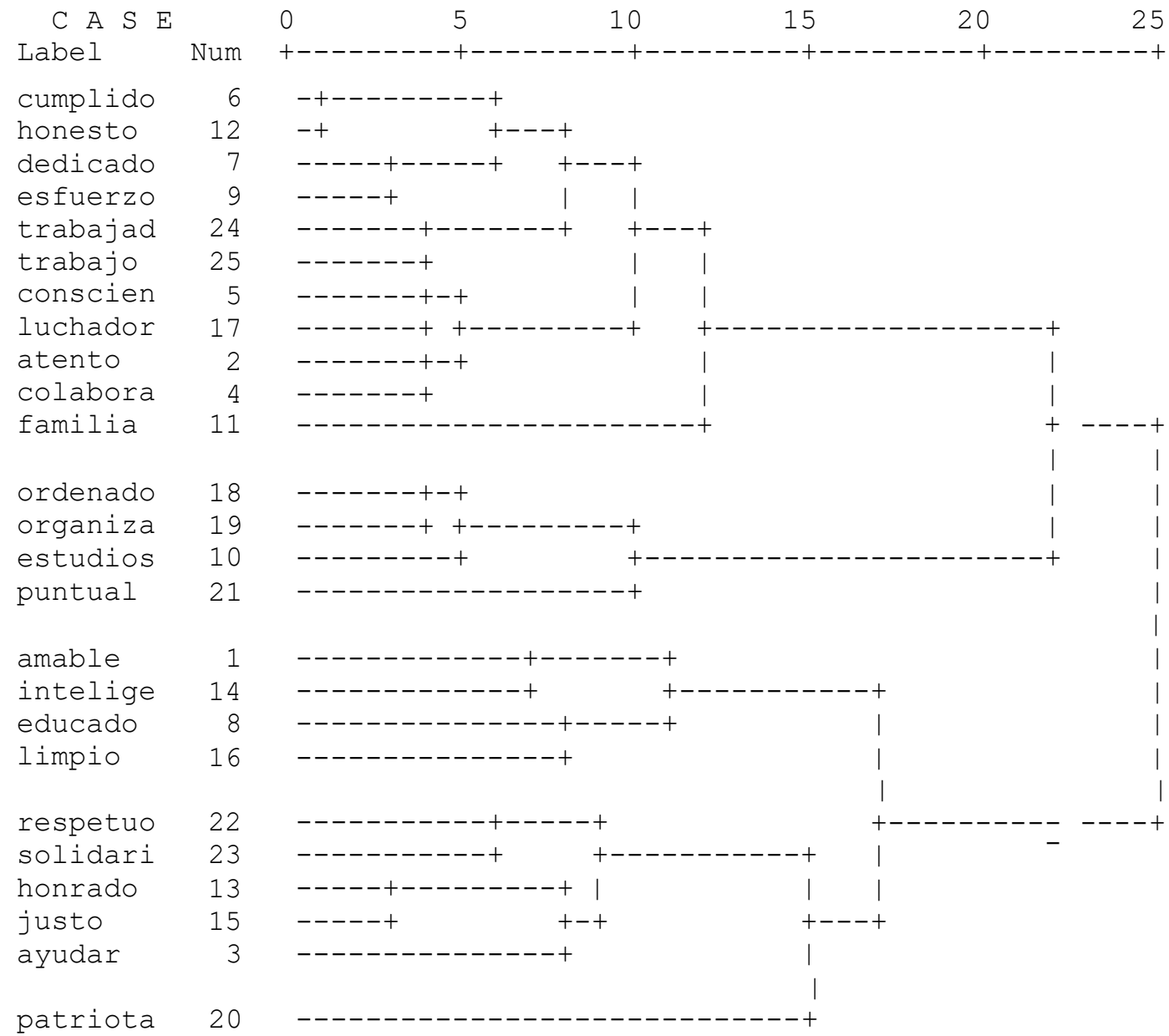


A partir de los resultados obtenidos en el análisis de cluster jerárquico para analizar la estructura común de los individuos de la palabra estímulo Europeo responsable se encontraron 2 grandes grupos que están más alejados entre sí, donde el grupo A se conforma por las palabras: dedicado, perseverante, emprendedor, exitoso, estudioso, comprometido, cumplido, ordenado, organizado, trabajador, trabajo, puntual, estudio, educación, educado, cultura, culto, inteligente, honesto, justo, respetuoso y consciente. El grupo B queda conformado por las siguientes palabras: amable, limpio, patriota. El grupo A puede dividirse en 2 grupos, donde A1 corresponde a: dedicado, perseverante, emprendedor, exitoso, estudioso, comprometido, cumplido, ordenado, organizado, trabajador, trabajo, puntual, estudio y A2 queda conformado por: educación, educado, cultura, culto, inteligente, honesto, justo, respetuoso y consciente. Las palabras más cercanas entre sí del grupo A son dedicado-perseverante, emprendedor-exitoso, comprometido-cumplido, ordenado-organizado. Del grupo B son: amable-limpio. Se observa que la palabra patriota es la más alejada del resto de palabras del grupo B. (ver tabla 15)

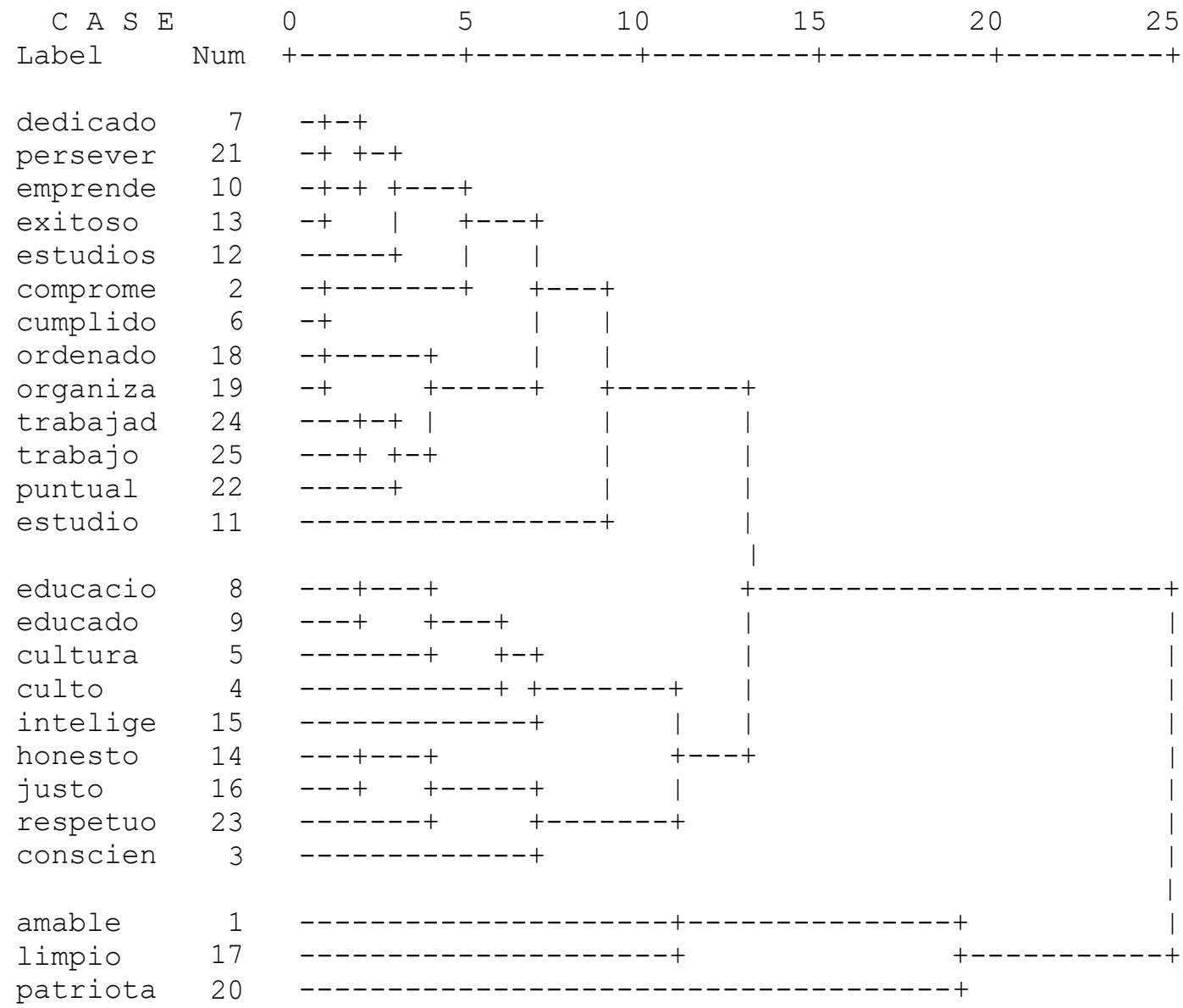


A partir de los resultados obtenidos en el análisis de cluster jerárquico para analizar la estructura común de los individuos de la palabra estímulo Yo irresponsable se encontraron 2 grandes grupos que están más alejados entre sí, donde el grupo A se conforma por las palabras: desordenado, impuntual, haragán, perezoso, mediocre, dejado, descuidado, despreocupado, desorganizado, distraído, desinteresado, olvidadizo, conformista, incumplido, inconsciente, indiferente, fracaso. El grupo B queda conformado por: incapaz, negativo, egoísta, malo, sucio, deshonesto, irrespetuoso y mentiroso. El grupo A puede dividirse en dos grupos, A1 que está conformado por: desordenado, impuntual, haragán, perezoso, mediocre, dejado, descuidado, despreocupado, desorganizado, distraído, desinteresado, olvidadizo y el grupo A2 conformado por: conformista, incumplido, inconsciente, indiferente, fracaso. El grupo B puede dividirse en B1 conformado por: incapaz, negativo y egoísta. B2 compuesto por: malo, sucio, deshonesto, irrespetuoso y mentiroso. Se puede observar que las palabras más cercanas en el grupo A son: desordenado-impuntual, haragán-perezoso y en el grupo B son: deshonesto-irrespetuoso. (ver tabla 16)

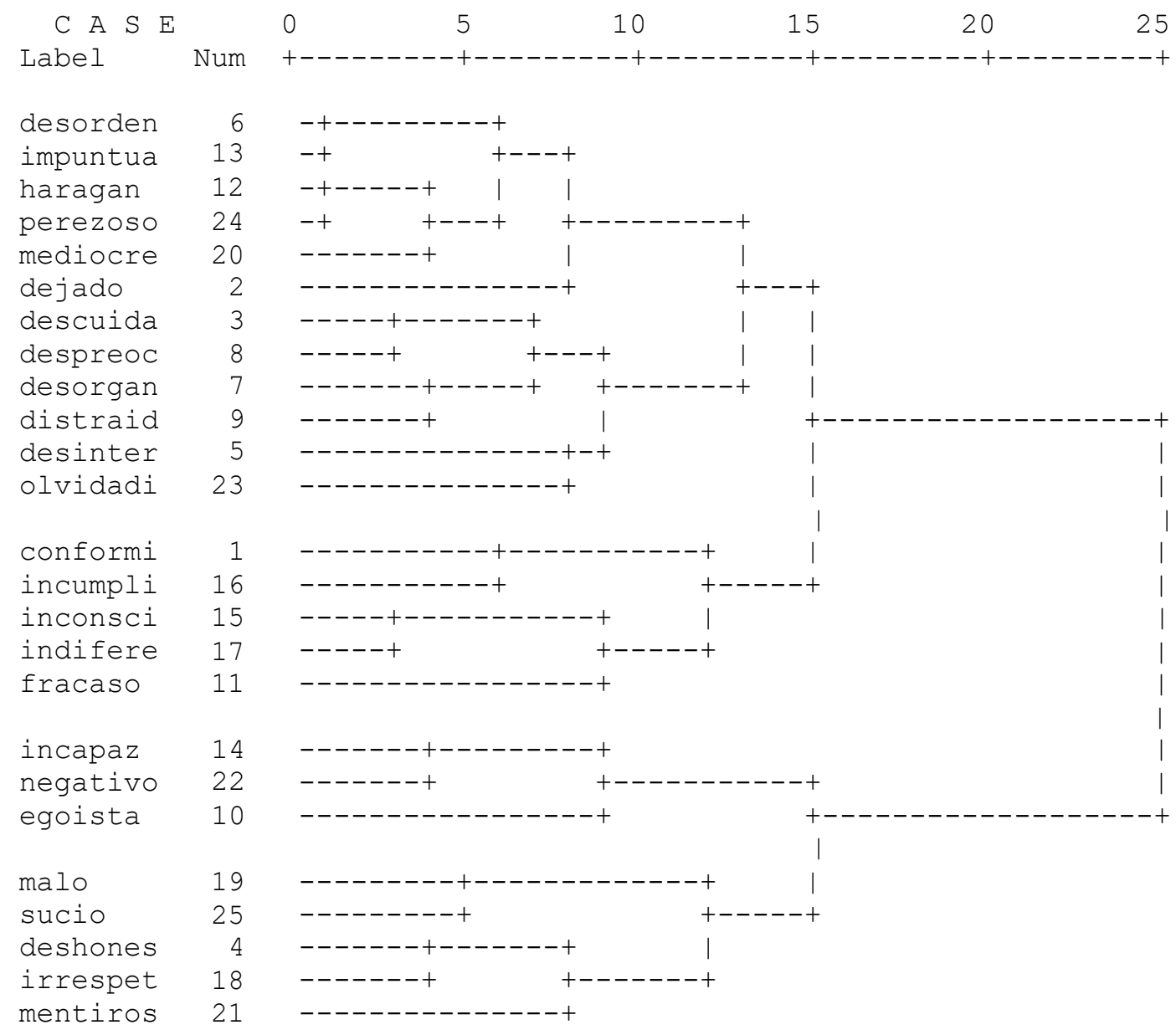


A partir de los resultados obtenidos en el análisis de cluster jerárquico para analizar la estructura común de los individuos de la palabra estímulo Guatemalteco irresponsable se encontraron 2 grandes grupos que están más alejados entre sí, donde el grupo A se conforma por las palabras: dejado, perezoso, despreocupado, descuidado, desorganizado, desinteresado, incumplido, haragán, impuntual, vago, conformista, desordenado. El grupo B queda conformado por las palabras: desempleado, ignorante, irrespetuoso, maleducado, deshonesto, inconsciente, sucio, borracho, ladrón, egoísta, violencia, corrupto y mentiroso. El grupo A puede dividirse en 2 grupos, A1 que está conformado por: dejado, perezoso, despreocupado, descuidado, desorganizado, desinteresado, incumplido, haragán, impuntual, vago y el grupo A2 conformado por: conformista y desordenado. El grupo B puede dividirse en B1 conformado por: desempleado e ignorante, B2 compuesto por: irrespetuoso, maleducado, deshonesto, inconsciente y sucio. B3 conformado por: borracho, ladrón, egoísta, violencia, corrupto y mentiroso. Las palabras más cercanas entre sí del grupo A son: dejado-perezoso y del grupo B son: irrespetuoso-maleducado. Las palabras más lejanas entre sí son desempleado e ignorante. (ver tabla 17)

TABLA 17

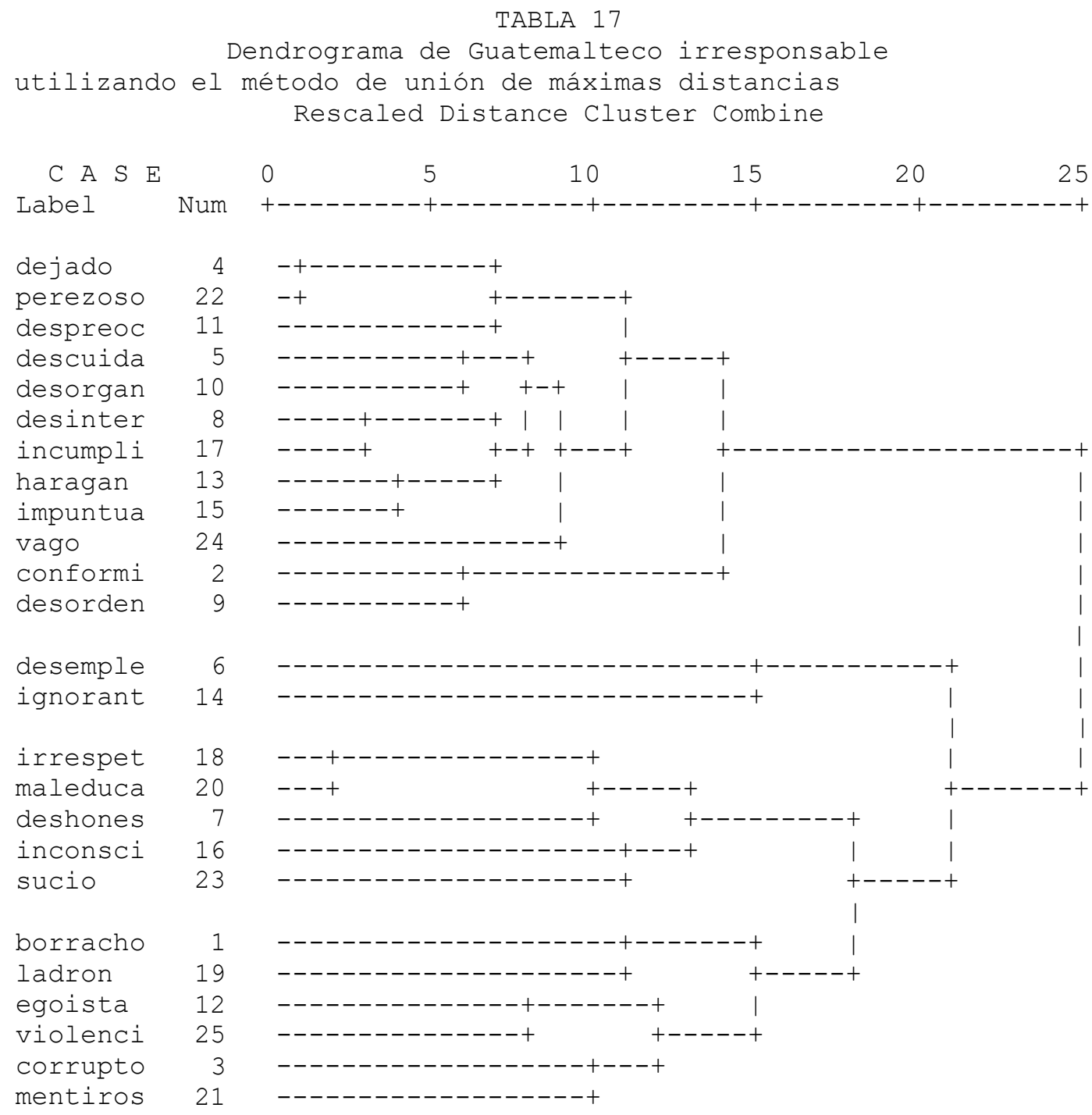


analizar la estructura común de los individuos de la palabra estímulo Europeo irresponsable se encontraron 2 grandes grupos que están más alejados entre sí, donde el grupo A se conforma por las palabras: descuidado, despreocupado, dejado, incumplido, perezoso, haragán, impuntual, conformista, borracho, drogas, desordenado, desorganizado, vago, desinteresado, indiferente, inconsciente, deshonesto, mentiroso, irrespetuoso y corrupto. El grupo B se conforma por: ignorante, sucio, egoísta, racista y fiestero. El grupo A puede dividirse en A1 que está conformado por: descuidado, despreocupado, dejado, incumplido, perezoso, haragán, impuntual, conformista y el grupo A2 compuesto por borracho, drogas, desordenado, desorganizado, vago, desinteresado, indiferente, inconsciente, deshonesto, mentiroso, irrespetuoso y corrupto. El grupo B puede dividirse en B1 compuesto por: ignorante y sucio. El grupo B2 conformado por: egoísta, racista y fiestero. Las palabras más cercanas entre sí del grupo A son: descuidado-despreocupado, incumplido-perezoso. Del grupo B son: egoístaracista. La palabra fiestero es la palabra más alejada que las de su grupo (ver tabla 18)

TABLA 18

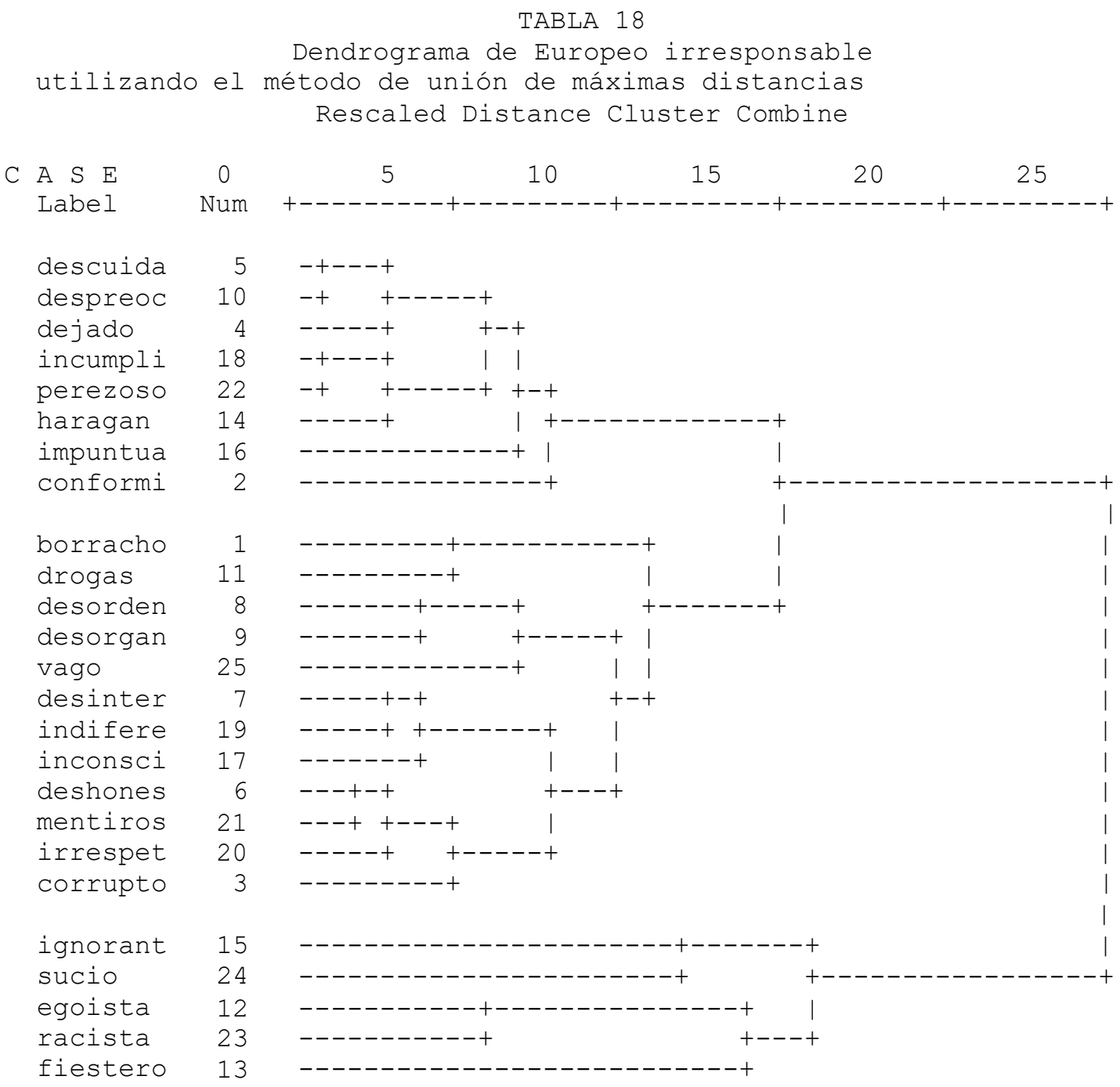


A partir de los resultados obtenidos en el análisis factorial exploratorio a través del método de análisis de componentes principales para analizar las posiciones individuales en el campo de la representación para Yo responsable, se obtuvo un índice de adecuación muestral de Kaiser-Meyer-Olkin de 0,921 y la prueba de esfericidad de Bartlett (chi cuadrada $=8793,174 ; \mathrm{gl}=1225 ; \mathrm{Sig} .=0,000$ ) que mostraron valores que permiten la utilización del análisis factorial.

Se obtuvieron 3 factores que explicaron el 51,619\% de la varianza y una confiabilidad total de 0,814 . El primer factor que se denominó "Lo que no es ser responsable" explicó el 25,635\% de la varianza, su confiabilidad fue de 0,95. El segundo factor denominado "Responsabilidad Normativa" explicó el 16,118\% de la varianza y su confiabilidad fue de 0,911 . El tercer factor "Responsabilidad hacia otros" cargó con el 9,866\% de la varianza con una confiabilidad de 0,826. Haciendo un análisis posterior de la información obtenida en los resultados, se encontró que de las 25 palabras tomadas de la red semántica obtenida para definir Yo responsable el 80\% (20) de éstas cargaron en los factores 2 y 3 , los cuales en conjunto, permiten explicar el $25,98 \%$ de la varianza total de los datos. (ver tabla 19)

\begin{tabular}{|c|c|c|c|c|c|}
\hline \multicolumn{6}{|c|}{$\begin{array}{c}\text { TABLA } 19 \\
\text { ANÁLISIS FACTORIAL EXPLORATORIO } \\
\text { YO RESPONSABLE }\end{array}$} \\
\hline \multicolumn{3}{|c|}{ FACTOR } & $\begin{array}{c}\text { Lo que no es } \\
\text { ser } \\
\text { responsable }\end{array}$ & $\begin{array}{c}\text { Responsabilidad } \\
\text { Normativa }\end{array}$ & $\begin{array}{c}\text { Responsabilidad } \\
\text { hacia otros }\end{array}$ \\
\hline \multicolumn{3}{|c|}{ Valor propio } & 10,767 & 6,770 & 4,144 \\
\hline \begin{tabular}{l|l}
$\%$ varianza $=51,619$ & $\%$ \\
\end{tabular} & \multicolumn{2}{|c|}{$\%$ varianza explicada por factor } & 25,635 & 16,118 & 9,866 \\
\hline Confiabilidad total $=0,814$ & Confiabilid & d por factor & 0,95 & 0,911 & 0,826 \\
\hline \multicolumn{3}{|c|}{ Media de correlaciones } & 0,459 & 0,444 & 0,406 \\
\hline \multicolumn{3}{|c|}{ Media de cada factor } & 4,110 & 1,835 & 2,126 \\
\hline \multicolumn{3}{|c|}{ Desviación Estándar de cada factor } & 0,769 & 0,676 & 0,827 \\
\hline & Media & D.E. & & & \\
\hline descuidado & 4,06 & 1,065 & ,821 & & \\
\hline desorganizado & 3,90 & 1,176 & ,776 & & \\
\hline mediocre & 4,33 & ,997 & ,754 & & \\
\hline perezoso & 4,03 & 1,190 &, 751 & & \\
\hline deshonesto & 4,32 & 1,064 &, 750 & & \\
\hline haragán & 4,06 & 1,183 &, 749 & & \\
\hline desordenado & 3,94 & 1,176 &, 745 & & \\
\hline despreocupado & 3,97 & 1,085 & ,739 & & \\
\hline impuntual & 3,99 & 1,258 &, 737 & & \\
\hline incumplido & 4,13 & 1,227 &, 735 & & \\
\hline inconsciente & 4,16 & 1,052 & ,730 & & \\
\hline dejado & 3,99 & 1,161 &, 723 & & \\
\hline conformista & 3,89 & 1,191 & ,689 & & \\
\hline irrespetuoso & 4,32 & 1,050 & 671 & & \\
\hline olvidadizo & 3,60 & 1,218 & ,662 & & \\
\hline fracaso & 4,26 & 1,033 & ,591 & & \\
\hline
\end{tabular}




\begin{tabular}{|l|r|r|r|r|r|}
\hline negativo & 4,22 &, 989 &, 589 & & \\
\hline mentiroso & 4,20 & 1,162 &, 587 & & \\
\hline malo & 4,41 &, 912 &, 580 & & \\
\hline sucio & 4,43 &, 899 &, 522 & & \\
\hline desinteresado & 4,19 & 1,110 &, 496 & & \\
\hline egoísta & 4,05 &, 985 &, 402 & & \\
\hline cumplido & 1,61 &, 932 & &, 758 & \\
\hline comprometido & 1,69 &, 942 & &, 749 & \\
\hline disciplina & 1,79 &, 904 & &, 718 & \\
\hline trabajador & 1,65 &, 896 & &, 705 & \\
\hline organizado & 1,86 &, 993 & &, 705 & \\
\hline dedicado & 1,80 &, 985 & &, 703 & \\
\hline puntual & 1,72 & 1,004 & &, 690 & \\
\hline aplicado & 1,80 &, 912 & &, 667 & \\
\hline estudioso & 1,98 &, 988 & &, 629 & \\
\hline trabajo & 1,94 & 1,006 & &, 627 & \\
\hline ordenado & 2,02 &, 995 & &, 608 &, 667 \\
\hline estudio & 2,05 & 1,039 & &, 599 & \\
\hline consciente & 1,96 & 1,041 & &, 512 & \\
\hline limpio & 2,25 & 1,284 & & & \\
\hline inteligente & 2,37 & 1,277 & & & \\
\hline respetuoso & 2,10 & 1,209 & & & \\
\hline familia & 2,14 & 1,290 & & & \\
\hline atento & 2,18 & 1,112 & & & \\
\hline correcto & 2,03 & 1,072 & & & \\
\hline confiable & 1,81 &, 999 & & & \\
\hline & & & & \\
\hline
\end{tabular}

A partir de los resultados obtenidos en el análisis factorial exploratorio a través del método de análisis de componentes principales para analizar las posiciones individuales en el campo de la representación para Guatemalteco responsable, se obtuvo un índice de adecuación muestral de Kaiser-Meyer-Olkin de 0,953 y la prueba de esfericidad de Bartlett (chi cuadrada= 10092,514; gl= 1225; Sig.= 0,000) que mostraron valores que permiten la utilización del análisis factorial.

Se obtuvieron 3 factores que explicaron el 57,376\% de la varianza y una confiabilidad total de 0,89. El primer factor que se denominó "Lo que no es ser responsable" explicó el 37,565\% de la varianza, su confiabilidad fue de 0,964. El segundo factor denominado "Responsabilidad Normativa" explicó el 11,161\% de la varianza y su confiabilidad fue de 0,83 . El tercer factor "Responsabilidad hacia otros" cargó con el $8,651 \%$ de la varianza y su confiabilidad fue de 0,754 . Haciendo un análisis posterior de la información obtenida en los resultados, se encontró que de las 25 palabras tomadas de la red semántica obtenida para definir Guatemalteco responsable el $44 \%$ (11) de éstas cargaron en los factores 2 y 3 , los cuales en conjunto, permiten explicar el 19,81\% de la varianza total de los datos. (ver tabla 20) 


\begin{tabular}{|c|c|c|c|c|c|}
\hline \multicolumn{6}{|c|}{$\begin{array}{c}\text { TABLA } 20 \\
\text { ANÁLISIS FACTORIAL EXPLORATORIO } \\
\text { GUATEMALTECO RESPONSABLE }\end{array}$} \\
\hline \multicolumn{3}{|c|}{ FACTOR } & $\begin{array}{c}\text { Lo que no es } \\
\text { ser } \\
\text { responsable }\end{array}$ & $\begin{array}{c}\text { Responsabilidad } \\
\text { Normativa }\end{array}$ & $\begin{array}{c}\text { Responsabilidad } \\
\text { hacia otros }\end{array}$ \\
\hline \multicolumn{3}{|c|}{ Valor propio } & 12,021 & 3,572 & 2,768 \\
\hline$\%$ varianza $=57,376 \quad \%$ & \multicolumn{2}{|c|}{$\%$ varianza explicada por factor } & 37,565 & 11,161 & 8,651 \\
\hline Confiabilidad total $=0,89$ & Confiabilid & d por factor & 0,964 & 0,83 & 0,754 \\
\hline \multicolumn{3}{|c|}{ Media de correlaciones } & 0,561 & 0,45 & 0,382 \\
\hline \multicolumn{3}{|c|}{ Media de cada factor } & 3,683 & 2,182 & 2,651 \\
\hline \multicolumn{3}{|c|}{ Desviación Estándar de cada factor } & 1,000 & 0,822 & 0,886 \\
\hline & Media & D.E. & & & \\
\hline perezoso & 3,57 & 1,357 &, 810 & & \\
\hline dejado & 3,64 & 1,301 & ,806 & & \\
\hline corrupto & 3,56 & 1,546 & ,797 & & \\
\hline descuidado & 3,71 & 1,247 & ,796 & & \\
\hline deshonesto & 3,81 & 1,245 & ,790 & & \\
\hline desorganizado & 3,65 & 1,256 & ,785 & & \\
\hline conformista & 3,35 & 1,377 & ,779 & & \\
\hline vago & 3,81 & 1,413 & ,777 & & \\
\hline haragán & 3,67 & 1,361 &, 774 & & \\
\hline desordenado & 3,64 & 1,298 &, 773 & & \\
\hline impuntual & 3,40 & 1,495 & ,765 & & \\
\hline maleducado & 3,86 & 1,197 &, 733 & & \\
\hline ignorante & 3,81 & 1,266 &, 720 & & \\
\hline despreocupado & 3,51 & 1,308 &, 720 & & \\
\hline sucio & 3,89 & 1,208 & ,704 & & \\
\hline desempleado & 3,53 & 1,417 & ,704 & & \\
\hline egoísta & 3,74 & 1,156 & ,704 & & \\
\hline inconsciente & 3,83 & 1,130 & ,691 & & \\
\hline ladrón & 3,87 & 1,451 & ,685 & & \\
\hline mentiroso & 3,87 & 1,184 & 670 & & \\
\hline desinteresado & 3,64 & 1,248 & ,664 & & \\
\hline esfuerzo & 2,22 & 1,126 & & ,809 & \\
\hline dedicado & 2,22 & 1,126 & & ,697 & \\
\hline trabajo & 2,17 & 1,083 & & ,686 & \\
\hline trabajador & 1,82 & 1,000 & & 655 & \\
\hline luchador & 2,14 & 1,199 & & ,631 & \\
\hline ordenado & 2,54 & 1,168 & & ,626 & \\
\hline amable & 2,48 & 1,274 & & & ,756 \\
\hline inteligente & 2,59 & 1,142 & & &, 725 \\
\hline educado & 2,58 & 1,228 & & &, 707 \\
\hline limpio & 2,71 & 1,283 & & &, 655 \\
\hline
\end{tabular}




\begin{tabular}{|l|r|r|r|r|r|}
\hline patriota & 2,89 & 1,307 & & &, 550 \\
\hline
\end{tabular}

A partir de los resultados obtenidos en el análisis factorial exploratorio a través del método de análisis de componentes principales para analizar las posiciones individuales en el campo de la representación para Europeo responsable, se obtuvo un índice de adecuación muestral de Kaiser-Meyer-Olkin de 0,936 y la prueba de esfericidad de Bartlett (chi cuadrada $=10120,167$; gl= 1225; Sig.= 0,000) que mostraron valores que permiten la utilización del análisis factorial.

Se obtuvieron 3 factores que explicaron el 58,834\% de la varianza y una confiabilidad total de 0,881 . El primer factor que se denominó "Lo que no es ser responsable" explicó el 36,614\% de la varianza, su confiabilidad fue de 0,959. El segundo factor denominado "Responsabilidad Normativa" explicó el 14,737\% de la varianza y su confiabilidad fue de 0,881 . El tercer factor "Responsabilidad hacia otros" cargó con el 7,483\% de la varianza y su confiabilidad fue de 0,67. Haciendo un análisis posterior de la información obtenida en los resultados, se encontró que de las 25 palabras tomadas de la red semántica obtenida para definir Europeo responsable el 44\% (11) de éstas cargaron en los factores 2 y 3 , los cuales en conjunto, permiten explicar el $22,22 \%$ de la varianza total de los datos. (ver tabla 21 )

\begin{tabular}{|c|c|c|c|c|c|}
\hline \multicolumn{6}{|c|}{$\begin{array}{c}\text { TABLA } 21 \\
\text { ANÁLISIS FACTORIAL EXPLORATORIO } \\
\text { EUROPEO RESPONSABLE }\end{array}$} \\
\hline \multicolumn{3}{|c|}{ FACTOR } & $\begin{array}{l}\text { Lo que no es } \\
\text { ser } \\
\text { responsable }\end{array}$ & $\begin{array}{c}\text { Responsabilidad } \\
\text { Normativa }\end{array}$ & $\begin{array}{c}\text { Responsabilidad } \\
\text { hacia otros }\end{array}$ \\
\hline \multicolumn{3}{|c|}{ Valor propio } & 10,618 & 4,274 & 2,170 \\
\hline \begin{tabular}{l|l|}
$\%$ varianza $=58,834$ & $\%$ \\
\end{tabular} & \multicolumn{2}{|c|}{$\%$ varianza explicada por factor } & 36,614 & 14,737 & 7,483 \\
\hline Confiabilidad total $=0,881$ & Confiabilid & d por factor & 0,959 & 0,881 & 0,670 \\
\hline \multicolumn{3}{|c|}{ Media de correlaciones } & 0,569 & 0,516 & 0,339 \\
\hline \multicolumn{3}{|c|}{ Media de cada factor } & 3,929 & 1,852 & 2,537 \\
\hline \multicolumn{3}{|c|}{ Desviación Estándar de cada factor } & 0,90 & 0,761 & 0,907 \\
\hline & Media & D.E. & & & \\
\hline perezoso & 3,95 & 1,131 & ,841 & & \\
\hline dejado & 3,91 & 1,146 & 820 & & \\
\hline deshonesto & 3,96 & 1,126 & ,809 & & \\
\hline despreocupado & 3,84 & 1,165 & ,806 & & \\
\hline descuidado & 3,80 & 1,210 & ,802 & & \\
\hline desordenado & 3,79 & 1,212 & 802 & & \\
\hline haragán & 3,96 & 1,201 & ,799 & & \\
\hline incumplido & 4,04 & 1,165 & ,798 & & \\
\hline mentiroso & 3,91 & 1,167 & ,796 & & \\
\hline corrupto & 4,07 & 1,167 & ,769 & & \\
\hline impuntual & 4,09 & 1,170 & ,763 & & \\
\hline desorganizado & 3,92 & 1,169 &, 762 & & \\
\hline inconsciente & 3,88 & 1,168 & ,749 & & \\
\hline irrespetuoso & 3,98 & 1,112 & ,740 & & \\
\hline
\end{tabular}




\begin{tabular}{|l|r|r|r|r|r|}
\hline conformista & 3,96 & 1,081 &, 706 & & \\
\hline vago & 3,99 & 1,250 &, 685 & & \\
\hline ignorante & 4,09 & 1,184 &, 639 & & \\
\hline desinteresado & 3,60 & 1,220 &, 546 & & \\
\hline cumplido & 1,85 &, 985 & &, 763 & \\
\hline puntual & 1,62 &, 933 & &, 749 & \\
\hline dedicado & 1,89 &, 986 & &, 728 & \\
\hline trabajador & 1,80 & 1,017 & &, 727 & \\
\hline organizado & 1,84 &, 981 & &, 727 & \\
\hline comprometido & 1,98 & 1,038 & &, 710 &, 763 \\
\hline ordenado & 1,99 & 1,030 & &, 689 &, 687 \\
\hline amable & 2,68 & 1,262 & & &, 650 \\
\hline patriota & 2,53 & 1,347 & & & \\
\hline inteligente & 2,22 & 1,183 & & & \\
\hline limpio & 2,72 & 1,322 & & & \\
\hline
\end{tabular}

A partir de los resultados obtenidos en el análisis factorial exploratorio a través del método de análisis de componentes principales para analizar las posiciones individuales en el campo de la representación para Yo irresponsable, se obtuvo un índice de adecuación muestral de Kaiser-Meyer-Olkin de 0,939 y la prueba de esfericidad de Bartlett (chi cuadrada=9038,408; gl= 1225; Sig.= 0,000) que mostraron valores que permiten la utilización del análisis factorial.

Se obtuvieron 3 factores que explicaron el $54,157 \%$ de la varianza y una confiabilidad total de 0,804. El primer factor que se denominó "Lo que no es ser irresponsable" explicó el 29,133\% de la varianza, su confiabilidad fue de 0,945. El segundo factor denominado "Irresponsabilidad Normativa" explicó el 15,012\% de la varianza y su confiabilidad fue de 0,865 . El tercer factor "Irresponsabilidad hacia otros" cargó con el 10,011\% de la varianza y su confiabilidad fue de 0,847 . Haciendo un análisis posterior de la información obtenida en los resultados, se encontró que de las 25 palabras tomadas de la red semántica obtenida para definir Yo irresponsable el 56\% (14) de éstas cargaron en los factores 2 y 3 , los cuales en conjunto, permiten explicar el $25,02 \%$ de la varianza total de los datos. (ver tabla 22) 


\begin{tabular}{|c|c|c|c|c|c|}
\hline \multicolumn{6}{|c|}{$\begin{array}{c}\text { TABLA } 22 \\
\text { ANÁLISIS FACTORIAL EXPLORATORIO }\end{array}$} \\
\hline & & ACTOR & $\begin{array}{c}\text { Lo que no es } \\
\text { ser } \\
\text { irresponsabl } \\
e\end{array}$ & $\begin{array}{l}\text { Irresponsabilidad } \\
\text { Normativa }\end{array}$ & $\begin{array}{l}\text { Irresponsabilidad } \\
\text { hacia otros }\end{array}$ \\
\hline \multicolumn{3}{|c|}{ Valor propio } & 9,323 & 4,804 & 3,204 \\
\hline$\%$ varianza $=54,157$ & \multicolumn{2}{|c|}{$\%$ varianza explicada por factor } & 29,133 & 15,012 & 10,011 \\
\hline \multicolumn{3}{|c|}{\begin{tabular}{|l|l|} 
Confiabilidad total $=0,804$ & Confiabilidad por factor \\
\end{tabular}} & 0,945 & 0,865 & 0,847 \\
\hline \multirow{2}{*}{\multicolumn{3}{|c|}{$\begin{array}{c}\text { Media de correlaciones } \\
\text { Media de cada factor }\end{array}$}} & 0,489 & 0,418 & 0,524 \\
\hline & & & 3,761 & 2,425 & 3,082 \\
\hline \multicolumn{3}{|c|}{ Desviación Estándar de cada factor } & 0,94 & 0,929 & 1,119 \\
\hline & Media & D.E. & & & \\
\hline trabajador & 3,81 & 1,390 & ,768 & & \\
\hline cumplido & 3,81 & 1,420 & ,767 & & \\
\hline honesto & 3,80 & 1,284 & ,756 & & \\
\hline esfuerzo & 3,58 & 1,422 & ,756 & & \\
\hline dedicado & 3,85 & 1,336 & ,752 & & \\
\hline cuidadoso & 3,79 & 1,257 & ,743 & & \\
\hline limpio & 3,79 & 1,276 & ,740 & & \\
\hline estudioso & 3,75 & 1,333 & ,721 & & \\
\hline trabajo & 3,64 & 1,290 & ,716 & & \\
\hline ordenado & 3,87 & 1,258 & ,700 & & \\
\hline organizado & 3,95 & 1,279 & ,685 & & \\
\hline disciplina & 3,62 & 1,378 & ,683 & & \\
\hline tareas & 3,33 & 1,414 &, 680 & & \\
\hline respetuoso & 3,88 & 1,235 & 678 & & \\
\hline familia & 3,73 & 1,311 & ,668 & & \\
\hline consciente & 3,80 & 1,184 & ,647 & & \\
\hline confiable & 3,84 & 1,240 & ,609 & & \\
\hline universidad & 3,85 & 1,187 &, 600 & & \\
\hline haragán & 1,99 & 1,290 & & ,723 & \\
\hline conformista & 2,70 & 1,233 & & ,695 & \\
\hline dejado & 2,20 & 1,350 & & ,688 & \\
\hline despreocupado & 2,39 & 1,310 & & 687 & \\
\hline indiferente & 2,49 & 1,292 & & ,685 & \\
\hline incumplido & 2,30 & 1,470 & & ,680 & \\
\hline fracaso & 2,62 & 1,467 & & ,636 & \\
\hline desinteresado & 2,55 & 1,335 & & ,577 & \\
\hline olvidadizo & 2,57 & 1,293 & & ,529 & \\
\hline malo & 3,23 & 1,454 & & &, 824 \\
\hline sucio & 3,08 & 1,453 & & & ,737 \\
\hline egoísta & 3,31 & 1,288 & & & ,734 \\
\hline deshonesto & 2,81 & 1,482 & & & ,721 \\
\hline
\end{tabular}




\begin{tabular}{|l|r|r|l|l|r|}
\hline irrespetuoso & 2,98 & 1,421 & & &, 700 \\
\hline
\end{tabular}

A partir de los resultados obtenidos en el análisis factorial exploratorio a través del método de análisis de componentes principales para analizar las posiciones individuales en el campo de la representación para Guatemalteco irresponsable, se obtuvo un índice de adecuación muestral de Kaiser-Meyer-Olkin de 0,922 y la prueba de esfericidad de Bartlett (chi cuadrada= 9084,457; gl= 1225; Sig.=0,000) que mostraron valores que permiten la utilización del análisis factorial.

Se obtuvieron 3 factores que explicaron el 53,129\% de la varianza y una confiabilidad total de 0,902. El primer factor que se denominó "Lo que no es ser irresponsable" explicó el 32,172\% de la varianza, su confiabilidad fue de 0,964. El segundo factor denominado "Irresponsabilidad hacia otros" explicó el 11,274\% de la varianza y su confiabilidad fue de 0,87 . El tercer factor "Irresponsabilidad Normativa" cargó con el 9,683\% de la varianza y su confiabilidad fue de 0,852 . Haciendo un análisis posterior de la información obtenida en los resultados, se encontró que de las 25 palabras tomadas de la red semántica obtenida para definir Guatemalteco irresponsable el 68\% (17) de éstas cargaron en los factores 2 y 3, los cuales en conjunto, permiten explicar el 20,957\% de la varianza total de los datos. (ver tabla 23)

\begin{tabular}{|c|c|c|c|c|c|}
\hline & $\begin{array}{l}\text { JÁLISIS } \\
\text { GUATEI }\end{array}$ & $\begin{array}{r}\text { TAB } \\
\text { ACTOR } \\
\text { IALTEC }\end{array}$ & $\begin{array}{l}\text { LA } 23 \\
\text { AL EXPLO } \\
\text { IRRESPO }\end{array}$ & $\begin{array}{l}\text { RATORIO } \\
\text { ISABLE }\end{array}$ & \\
\hline & & $A C T O R$ & $\begin{array}{c}\text { Lo que no es } \\
\text { ser } \\
\text { irresponsabl } \\
e \\
\end{array}$ & $\begin{array}{l}\text { Irresponsabilidad } \\
\text { hacia otros }\end{array}$ & $\begin{array}{c}\text { Irresponsabilidad } \\
\text { Normativa }\end{array}$ \\
\hline & $\mathrm{Va}$ & or propio & 13,512 & 4,735 & 4,067 \\
\hline$\%$ varianza $=53,129 \%$ & anza explica & a por factor & 32,172 & 11,274 & 9,683 \\
\hline Confiabilidad total $=0,902$ & Confiabilid & d por factor & 0,964 & 0,87 & 0,852 \\
\hline & lia de corr & elaciones & 0,523 & 0,426 & 0,423 \\
\hline & Iedia de ca & da factor & 3,89 & 2,422 & 2,031 \\
\hline Desviación Es & ndar de ca & la factor & 0,877 & 0,901 & 0,783 \\
\hline & Media & D.E. & & & \\
\hline dedicado & 3,91 & 1,213 & ,791 & & \\
\hline honesto & 3,89 & 1,207 & ,788 & & \\
\hline honrado & 4,00 & 1,141 &, 773 & & \\
\hline trabajador & 3,78 & 1,267 & ,768 & & \\
\hline cumplido & 3,92 & 1,238 & ,767 & & \\
\hline trabajo & 3,84 & 1,218 & ,766 & & \\
\hline consciente & 3,72 & 1,254 & ,761 & & \\
\hline organizado & 3,96 & 1,214 & ,759 & & \\
\hline estudioso & 3,98 & 1,146 & ,758 & & \\
\hline luchador & 3,91 & 1,199 & ,738 & & \\
\hline justo & 4,04 & 1,057 & ,737 & & \\
\hline ayudar & 3,90 & 1,140 & ,736 & & \\
\hline respetuoso & 3,84 & 1,111 & ,735 & & \\
\hline ordenado & 3,93 & 1,162 & ,734 & & \\
\hline
\end{tabular}




\begin{tabular}{|c|c|c|c|c|c|}
\hline esfuerzo & 3,82 & 1,239 & ,733 & & \\
\hline solidario & 4,07 & 1,095 & ,728 & & \\
\hline colaborador & 3,89 & 1,237 & ,698 & & \\
\hline puntual & 3,81 & 1,366 & 689 & & \\
\hline patriota & 3,91 & 1,278 & 689 & & \\
\hline limpio & 4,00 & 1,123 & 689 & & \\
\hline educado & 3,81 & 1,099 & ,684 & & \\
\hline atento & 3,92 & 1,097 & ,684 & & \\
\hline amable & 3,86 & 1,196 & 678 & & \\
\hline inteligente & 3,89 & 1,178 & ,663 & & \\
\hline familia & 3,64 & 1,329 & ,598 & & \\
\hline maleducado & 2,49 & 1,289 & & ,805 & \\
\hline irrespetuoso & 2,56 & 1,285 & &, 778 & \\
\hline egoísta & 2,56 & 1,309 & & ,776 & \\
\hline violencia & 2,35 & 1,337 & & ,662 & \\
\hline mentiroso & 2,24 & 1,258 & & ,659 & \\
\hline corrupto & 2,05 & 1,308 & & ,621 & \\
\hline deshonesto & 2,50 & 1,207 & & ,596 & \\
\hline ignorante & 2,76 & 1,274 & & ,586 & \\
\hline ladrón & 2,27 & 1,301 & &, 566 & \\
\hline dejado & 1,91 & 1,065 & & & ,731 \\
\hline perezoso & 1,86 & 1,033 & & & ,697 \\
\hline despreocupado & 2,25 & 1,181 & & & ,691 \\
\hline haragán & 1,80 & 1,061 & & & ,686 \\
\hline conformista & 2,32 & 1,245 & & & ,640 \\
\hline desorganizado & 2,09 & 1,084 & & &, 610 \\
\hline desordenado & 2,24 & 1,202 & & &, 610 \\
\hline impuntual & 1,79 & 1,052 & & &, 585 \\
\hline
\end{tabular}

A partir de los resultados obtenidos en el análisis factorial exploratorio a través del método de análisis de componentes principales para analizar las posiciones individuales en el campo de la representación para Europeo irresponsable, se obtuvo un índice de adecuación muestral de Kaiser-Meyer-Olkin de 0,932 y la prueba de esfericidad de Bartlett (chi cuadrada= 9082,407; gl= 1225; Sig.= 0,000) que mostraron valores que permiten la utilización del análisis factorial.

Se obtuvieron 3 factores que explicaron el 55,852\% de la varianza y una confiabilidad total de 0,897. El primer factor que se denominó "Lo que no es ser irresponsable" explicó el 34,9\% de la varianza, su confiabilidad fue de 0,966. El segundo factor denominado "Irresponsabilidad Normativa" explicó el 16,185\% de la varianza y su confiabilidad fue de 0,904. El tercer factor "Irresponsabilidad hacia otros" cargó con el 4,767\% de la varianza y su confiabilidad fue de 0,67 . Haciendo un análisis posterior de la información obtenida en los resultados, se encontró que de las 25 palabras tomadas de la red semántica obtenida para definir Europeo irresponsable el 
$56 \%$ (14) de éstas cargaron en los factores 2 y 3 , los cuales en conjunto, permiten explicar el 20,952\% de la varianza total de los datos. (ver tabla 24)

\begin{tabular}{|c|c|c|c|c|c|}
\hline & $\begin{array}{c}\text { ÁLISIS } \\
\text { EUR }\end{array}$ & $\begin{array}{r}\text { TAB } \\
\text { ACTOR } \\
\text { OPEO IR }\end{array}$ & $\begin{array}{l}\text { LA } 24 \\
\text { AL EXPLC } \\
\text { RESPONS }\end{array}$ & $\begin{array}{l}\text { RATORIO } \\
\text { BLE }\end{array}$ & \\
\hline & & $A C T O R$ & $\begin{array}{c}\text { Lo que no es } \\
\text { ser } \\
\text { irresponsabl } \\
e \\
\end{array}$ & $\begin{array}{c}\text { Irresponsabilidad } \\
\text { Normativa }\end{array}$ & $\begin{array}{l}\text { Irresponsabilidad } \\
\text { hacia otros }\end{array}$ \\
\hline & Val & or propio & 13,611 & 6,312 & 1,859 \\
\hline$\%$ varianza $=55,852 \quad \%$ & anza explicac & a por factor & 34,9 & 16,185 & 4,767 \\
\hline Confiabilidad total $=0,897$ & Confiabilid & d por factor & 0,966 & 0,904 & 0,67 \\
\hline & ia de corr & elaciones & 0,533 & 0,443 & 0,506 \\
\hline & edia de ca & da factor & 3,788 & 2,25 & 2,813 \\
\hline Desviación E & idar de ca & da factor & 0,933 & 0,86 & 1,208 \\
\hline & Media & D.E. & & & \\
\hline cumplido & 3,88 & 1,272 & ,813 & & \\
\hline trabajador & 3,73 & 1,259 & ,804 & & \\
\hline exitoso & 3,80 & 1,326 & ,799 & & \\
\hline educación & 3,55 & 1,374 & ,783 & & \\
\hline dedicado & 3,81 & 1,252 & ,779 & & \\
\hline comprometido & 3,77 & 1,283 & ,777 & & \\
\hline perseverante & 3,78 & 1,310 & ,769 & & \\
\hline estudioso & 3,77 & 1,218 & ,768 & & \\
\hline trabajo & 3,78 & 1,322 & ,761 & & \\
\hline educado & 3,76 & 1,199 & ,757 & & \\
\hline estudio & 3,82 & 1,208 &, 754 & & \\
\hline emprendedor & 3,79 & 1,237 &, 741 & & \\
\hline puntual & 3,65 & 1,467 & ,734 & & \\
\hline ordenado & 3,87 & 1,181 &, 724 & & \\
\hline honesto & 3,83 & 1,209 & ,723 & & \\
\hline respetuoso & 3,85 & 1,140 & ,720 & & \\
\hline consciente & 3,72 & 1,285 & ,719 & & \\
\hline culto & 3,81 & 1,297 & ,714 & & \\
\hline organizado & 3,88 & 1,238 & ,706 & & \\
\hline inteligente & 3,74 & 1,226 & 679 & & \\
\hline justo & 3,83 & 1,159 &, 651 & & \\
\hline cultura & 3,63 & 1,283 & ,648 & & \\
\hline patriota & 3,82 & 1,302 & ,641 & & \\
\hline limpio & 3,88 & 1,239 & ,617 & & \\
\hline amable & 3,95 & 1,072 & ,588 & & \\
\hline dejado & 2,32 & 1,271 & &, 773 & \\
\hline incumplido & 2,05 & 1,155 & &, 751 & \\
\hline perezoso & 2,19 & 1,217 & &, 748 & \\
\hline
\end{tabular}




\begin{tabular}{|l|r|r|r|r|r|}
\hline despreocupado & 2,18 & 1,150 & &, 741 & \\
\hline desorganizado & 2,24 & 1,176 & &, 715 & \\
\hline descuidado & 2,18 & 1,182 & &, 697 & \\
\hline haragán & 2,03 & 1,206 & &, 695 & \\
\hline impuntual & 2,34 & 1,371 & &, 630 & \\
\hline desinteresado & 2,27 & 1,186 & &, 629 & \\
\hline vago & 2,38 & 1,334 & &, 626 & \\
\hline conformista & 2,43 & 1,324 & &, 586 & \\
\hline desordenado & 2,39 & 1,214 & & &, 749 \\
\hline racista & 2,92 & 1,458 & & & \\
\hline egoísta & 2,70 & 1,325 & & & \\
\hline
\end{tabular}

A partir de los resultados obtenidos en el análisis factorial exploratorio a través del método de análisis de componentes principales para analizar las posiciones individuales en el campo de la representación para el Autoconcepto de Responsabilidad, se obtuvo un índice de adecuación muestral de Kaiser-Meyer-Olkin de 0,888 y la prueba de esfericidad de Bartlett (chi cuadrada= 5829,432; gl= 1225; Sig.=0,000) que mostraron valores que permiten la utilización del análisis factorial. En cuanto a los análisis de correlación, se observó un buen nivel de independencia entre los reactivos, ya que las correlaciones obtenidas en ningún caso fueron mayores de 0,4 como puede verse en las medias de correlaciones de los factores.

Se obtuvieron 3 factores que explicaron el $42,874 \%$ de la varianza y una confiabilidad total de 0,766. El primer factor que se denominó "Irresponsabilidad Normativa" explicó el 17,991\% de la varianza, su confiabilidad fue de 0,867. Su media de 3,466 (con una desviación estándar de 0,709) indica que los sujetos tienen una posición neutral en este factor. El segundo factor denominado "Yo siendo responsable" explicó el 14,232\% de la varianza y su confiabilidad fue de 0,832 . Su media de 1,618 (con una desviación estándar de 0,545) indica que los sujetos tienden a considerarse altamente responsables, en su trabajo, con la familia, siendo confiables, responsables con la universidad, siendo conscientes, correctos, inteligentes, trabajadores y atentos. Lo anterior sugiere una tendencia mayor a la Responsabilidad hacia otros. El tercer factor "Irresponsabilidad hacia otros" cargó con el 10,651\% de la varianza y su confiabilidad fue de 0,752 . Su media de 4,03 (con una desviación estándar de 0,651) indica que los sujetos tienden a verse muy poco Irresponsables hacia otros. (ver tabla 25) 


\begin{tabular}{|c|c|c|c|c|c|}
\hline \multicolumn{6}{|c|}{$\begin{array}{c}\text { TABLA } 25 \\
\text { ANÁLISIS FACTORIAL EXPLORATORIO } \\
\text { AUTOCONCEPTO DE RESPONSABILIDAD }\end{array}$} \\
\hline \multicolumn{3}{|c|}{ FACTOR } & $\begin{array}{c}\text { Irresponsabilidad } \\
\text { Normativa } \\
\end{array}$ & $\begin{array}{c}\text { Yo siendo } \\
\text { responsable }\end{array}$ & $\begin{array}{c}\text { Irresponsabilidad } \\
\text { hacia otros }\end{array}$ \\
\hline \multicolumn{3}{|c|}{ Valor propio } & 5,038 & 3,985 & 2,982 \\
\hline$\%$ varianza $=42,874$ & \multicolumn{2}{|c|}{$\%$ varianza explicada por factor } & 17,991 & 14,232 & 10,651 \\
\hline Confiabilidad total $=0,766$ & Confiabilid & por factor & 0,867 & 0,832 & 0,752 \\
\hline \multicolumn{3}{|c|}{ Media de correlaciones } & 0,355 & 0,358 & 0,309 \\
\hline \multicolumn{3}{|c|}{ Media de cada factor } & 3,466 & 1,618 & 4,03 \\
\hline \multicolumn{3}{|c|}{ Desviación Estándar de cada factor } & 0,709 & 0,545 & 0,651 \\
\hline & Media & D.E. & & & \\
\hline desorganizado & 3,66 & 1,080 & 694 & & \\
\hline dejado & 3,51 & 1,073 & ,686 & & \\
\hline olvidadizo & 2,86 & 1,174 & ,684 & & \\
\hline descuidado & 3,75 & 1,033 & 682 & & \\
\hline desordenado & 3,55 & 1,101 & 645 & & \\
\hline perezoso & 3,50 & 1,070 & ,637 & & \\
\hline despreocupado & 3,51 & 1,140 & ,613 & & \\
\hline distraido & 2,67 & 1,124 & 601 & & \\
\hline desinteresado & 3,51 & 1,162 &, 583 & & \\
\hline haragan & 3,69 & 1,122 & ,582 & & \\
\hline indiferente & 3,59 & 1,079 &, 536 & & \\
\hline impuntual & 3,79 & 1,171 & ,461 & & \\
\hline trabajo & 1,81 & ,921 & & ,724 & \\
\hline familia & 1,46 &, 852 & & ,687 & \\
\hline confiable & 1,45 & ,776 & & ,674 & \\
\hline universidad & 1,65 & ,929 & & ,646 & \\
\hline consciente & 1,83 & ,928 & & ,623 & \\
\hline correcto & 1,58 & ,715 & & ,607 & \\
\hline inteligente & 1,46 & ,714 & & ,596 & \\
\hline trabajador & 1,67 &, 818 & & ,582 & \\
\hline atento & 1,65 &, 813 & &, 540 & \\
\hline malo & 4,20 & ,947 & & &, 752 \\
\hline sucio & 4,37 & ,886 & & &, 671 \\
\hline deshonesto & 4,27 & 1,063 & & & ,614 \\
\hline egoísta & 3,57 & 1,108 & & &, 592 \\
\hline mentiroso & 3,70 & 1,076 & & &, 535 \\
\hline incapaz & 4,30 & 1,036 & & &, 509 \\
\hline negativo & 3,81 & 1,053 & & & ,467 \\
\hline
\end{tabular}


Se calculó además el puntaje de cada participante con respecto a cada uno de los factores al promediar los reactivos que lo componen. Con base en esa puntuación se calculó la matriz de correlaciones entre factores. Se puede observar una correlación positiva significativa en el factor Irresponsabilidad hacia otros con Irresponsabilidad Normativa de 0,451 . Hay correlaciones negativas significativas entre los factores Yo siendo responsable con Irresponsabilidad Normativa de 0,375 y con Irresponsabilidad hacia otros de 0,354 (ver tabla 26)

\begin{tabular}{|c|c|c|}
\hline \multicolumn{3}{|c|}{$\begin{array}{c}\text { TABLA } 26 \\
\text { CORRELACIONES ENTRE FACTORES } \\
\text { AUTOCONCEPTO DE RESPONSABILIDAD }\end{array}$} \\
\hline & $\begin{array}{c}\text { Irresponsabilidad } \\
\text { Normativa }\end{array}$ & Yo siendo responsable \\
\hline \multicolumn{3}{|l|}{ Irresponsabilidad Normativa } \\
\hline Yo siendo responsable &,- 375 & \\
\hline Irresponsabilidad hacia otros & ,451" &,$- 354^{*}$ \\
\hline
\end{tabular}

A partir de los resultados obtenidos en el análisis factorial exploratorio a través del método de análisis de componentes principales para analizar las posiciones individuales en el campo de la representación para el Autoconcepto de Responsabilidad del Guatemalteco, se obtuvo un índice de adecuación muestral de Kaiser-Meyer-Olkin de 0,907 y la prueba de esfericidad de Bartlett (chi cuadrada $=5753,873$; gl= 1225;

Sig.= 0,000) que mostraron valores que permiten la utilización del análisis factorial. En cuanto a los análisis de correlación, se observó un buen nivel de independencia entre los reactivos, ya que las correlaciones obtenidas en ningún caso fueron mayores de 0,4 como puede verse en las medias de correlaciones de los factores.

Se obtuvieron 3 factores que explicaron el 51,521\% de la varianza y una confiabilidad total de 0,65. El primer factor que se denominó "Irresponsabilidad Normativa" explicó el 18,079\% de la varianza, su confiabilidad fue de 0,737. Su media de 2,513 (con una desviación estándar de 0,74 ) indica que los sujetos tienen una posición neutral en este factor. El segundo factor denominado "Responsabilidad Normativa" explicó el 17,494\% de la varianza y su confiabilidad fue de 0,737. Su media de 2,446 (con una desviación estándar de 0,719) indica una posición neutral en este factor. El tercer factor "Responsabilidad hacia otros" cargó con el 15,949\% de la varianza y su confiabilidad fue de 0,721 . Su media de 2,287 (con una desviación estándar de 0,781 ) indica que los sujetos tienden a ver al guatemalteco algo responsable hacia otros, a través de ayudar, siendo amables, solidarios y con la familia. (ver tabla 27) 


\begin{tabular}{|c|c|c|c|c|c|}
\hline \multicolumn{6}{|c|}{$\begin{array}{c}\text { TABLA } 27 \\
\text { ANÁLISIS FACTORIAL EXPLORATORIO AUTOCONCEPTO DE } \\
\text { RESPONSABILIDAD DEL GUATEMALTECO }\end{array}$} \\
\hline \multicolumn{3}{|c|}{ FACTOR } & $\begin{array}{l}\text { Irresponsabilidad } \\
\text { Normativa }\end{array}$ & $\begin{array}{l}\text { Responsabilida } \\
\text { d Normativa }\end{array}$ & $\begin{array}{l}\text { Responsabilidad } \\
\text { hacia otros }\end{array}$ \\
\hline \multicolumn{3}{|c|}{ Valor propio } & 2,531 & 2,449 & 2,233 \\
\hline \multicolumn{3}{|c|}{\begin{tabular}{c|c}
$\%$ varianza $=51,521$ & $\%$ varianza explicada por factor
\end{tabular}} & 18,079 & 17,494 & 15,949 \\
\hline \multirow{2}{*}{ Confiabilidad total $=0,65$} & & 0,737 & 0,737 & 0,721 \\
\hline & \multicolumn{2}{|c|}{$\begin{array}{l}\text { Confiabilidad por factor } \\
\text { Media de correlaciones }\end{array}$} & 0,359 & 0,358 & 0,392 \\
\hline \multicolumn{3}{|c|}{ Media de cada factor } & 2,513 & 2,446 & 2,287 \\
\hline \multicolumn{3}{|c|}{ Desviación Estándar de cada factor } & 0,74 & 0,719 & 0,781 \\
\hline & Media & D.E. & & & \\
\hline descuidado & 2,64 & 1,035 & ,784 & & \\
\hline conformista & 2,31 & 1,112 & ,777 & & \\
\hline desorganizado & 2,68 & 1,004 & 658 & & \\
\hline despreocupado & 2,31 & 1,020 & ,642 & & \\
\hline haragán & 2,62 & 1,127 & ,618 & & \\
\hline trabajo & 2,38 & 1,036 & & ,732 & \\
\hline trabajador & 2,27 & 1,065 & &, 725 & \\
\hline inteligente & 2,10 &, 915 & & ,636 & \\
\hline esfuerzo & 2,64 & 1,073 & & ,630 & \\
\hline consciente & 2,83 & 1,050 & &, 580 & \\
\hline amable & 2,14 & 1,050 & & & ,741 \\
\hline ayudar & 2,47 & 1,097 & & &, 713 \\
\hline solidario & 2,58 & 1,105 & & & 697 \\
\hline familia & 1,96 & ,980 & & & ,669 \\
\hline
\end{tabular}

Se calculó además el puntaje de cada participante con respecto a cada uno de los factores al promediar los reactivos que lo componen. Con base en esa puntuación se calculó la matriz de correlaciones entre factores. Se puede observar una correlación positiva significativa del factor Responsabilidad Normativa y Responsabilidad hacia otros de 0,532. Hay correlaciones negativas significativas entre los factores Irresponsabilidad Normativa con Responsabilidad Normativa de 0,178 y con Responsabilidad hacia otros de 0,119 (ver tabla 28)

\begin{tabular}{|c|c|c|}
\hline \multicolumn{3}{|c|}{$\begin{array}{c}\text { TABLA } 28 \\
\text { CORRELACIONES ENTRE FACTORES } \\
\text { AUTOCONCEPTO DE RESPONSABILIDAD DEL } \\
\text { GUATEMALTECO }\end{array}$} \\
\hline & $\begin{array}{c}\text { Irresponsabilidad } \\
\text { Normativa } \\
\end{array}$ & $\begin{array}{c}\text { Responsabilidad } \\
\text { Normativa }\end{array}$ \\
\hline \multicolumn{3}{|l|}{ Irresponsabilidad Normativa } \\
\hline Responsabilidad Normativa &,$- 178^{*}$ & \\
\hline Responsabilidad hacia otros &,- 119 &, 53 \\
\hline
\end{tabular}




\begin{tabular}{c} 
TABLA 28 \\
CORRELACIONES ENTRE FACTORES \\
AUTOCONCEPTO DE RESPONSABILIDAD DEL \\
GUATEMALTECO \\
\hline **. La correlación es significativa en el nivel del 0.01 (prueba de 2 colas). \\
*. La correlación es significativa en el nivel del 0.05 (prueba de 2 colas). \\
\hline
\end{tabular}

A partir de los resultados obtenidos en el análisis factorial exploratorio a través del método de análisis de componentes principales para analizar las posiciones individuales en el campo de la representación para el Autoconcepto de Responsabilidad del Europeo, se obtuvo un índice de adecuación muestral de Kaiser-Meyer-Olkin de 0,876 y la prueba de esfericidad de Bartlett (chi cuadrada= 5344,744; gl=1225; Sig.= 0,000) que mostraron valores que permiten la utilización del análisis factorial. En cuanto a los análisis de correlación, se observó un buen nivel de independencia entre los reactivos, ya que las correlaciones obtenidas en ningún caso fueron mayores de 0,4 como puede verse en las medias de correlaciones de los factores.

Se obtuvieron 3 factores que explicaron el $41,856 \%$ de la varianza y una confiabilidad total de 0,784 . El primer factor que se denominó "El europeo siendo responsable" explicó el 19,825\% de la varianza, su confiabilidad fue de 0,909. Su media de 2,084 (con una desviación estándar de 0,595) indica que los sujetos tienen a ver al europeo como altamente responsable, siendo dedicado, perseverante, estudioso, inteligente, organizado, comprometido, ordenado, exitoso, educado, honesto, emprendedor, siendo culto y teniendo cultura. El segundo factor denominado "Irresponsabilidad Normativa" explicó el 15,285\% de la varianza y su confiabilidad fue de 0,879 . Su media de 3,342 (con una desviación estándar de 0,656 ) indica una posición neutral en este factor. El tercer factor "Irresponsabilidad hacia otros" cargó con el $6,747 \%$ de la varianza y su confiabilidad fue de 0,618 . Su media de 2,43 (con una desviación estándar de 0,696) indica una posición neutral en este factor ( ver tabla 29).

\begin{tabular}{|c|c|c|c|c|c|}
\hline AUTOC & $\begin{array}{l}\text { NÁLISIS } \\
\text { JCEPTO }\end{array}$ & $\begin{array}{r}\text { TA } \\
\text { FACTOI } \\
\text { DE RESI }\end{array}$ & $\begin{array}{l}\text { BLA } 29 \\
\text { IAL EXPLOR } \\
\text { ONSABILIDA }\end{array}$ & $\begin{array}{l}\text { TORIO } \\
\text { DEL EURO }\end{array}$ & \\
\hline & & $A C T O R$ & $\begin{array}{c}\text { El europeo siendo } \\
\text { responsable }\end{array}$ & $\begin{array}{c}\text { Irresponsabilidad } \\
\text { Normativa }\end{array}$ & $\begin{array}{c}\text { Irresponsabilidad } \\
\text { hacia otros }\end{array}$ \\
\hline & Val & or propio & 7,137 & 5,503 & 2,429 \\
\hline$\%$ varianza $=41,856$ & nza explicas & a por factor & 19,825 & 15,285 & 6,747 \\
\hline Confiabilidad total $=0,784$ & Confiabilid & d por factor & 0,909 & 0,879 & 0,618 \\
\hline & a de corr & laciones & 0,363 & 0,359 & 0,245 \\
\hline & dia de ca & da factor & 2,084 & 3,342 & 2,43 \\
\hline Desviación Es & dar de ca & la factor & 0,595 & 0,656 & 0,696 \\
\hline & Media & D.E. & & & \\
\hline dedicado & 2,12 & ,924 & ,723 & & \\
\hline perseverante & 2,16 & ,883 & ,695 & & \\
\hline estudioso & 1,84 & ,897 & ,689 & & \\
\hline inteligente & 1,90 & ,889 & ,688 & & \\
\hline
\end{tabular}




\begin{tabular}{|c|c|c|c|c|c|}
\hline organizado & 1,99 & ,897 & ,677 & & \\
\hline comprometido & 2,16 & ,917 & ,653 & & \\
\hline ordenado & 2,12 & ,972 &, 652 & & \\
\hline exitoso & 2,01 & ,872 & ,639 & & \\
\hline educado & 1,89 & ,905 & ,637 & & \\
\hline honesto & 2,15 & ,967 &, 626 & & \\
\hline emprendedor & 2,05 & 1,013 & ,603 & & \\
\hline trabajo & 1,89 & ,927 & ,594 & & \\
\hline cumplido & 2,20 & ,947 &, 576 & & \\
\hline culto & 1,86 & ,955 &, 574 & & \\
\hline cultura & 1,79 & ,957 &, 530 & & \\
\hline consciente & 2,35 & ,982 & ,494 & & \\
\hline justo & 2,33 & ,971 & ,434 & & \\
\hline limpio & 2,70 & 1,187 & ,390 & & \\
\hline desorganizado & 3,39 & 1,019 & &, 750 & \\
\hline desordenado & 3,30 & 1,001 & & ,712 & \\
\hline incumplido & 3,50 & ,995 & & ,696 & \\
\hline impuntual & 3,67 & 1,130 & & ,683 & \\
\hline despreocupado & 3,10 & 1,095 & & ,665 & \\
\hline perezoso & 3,36 & 1,040 & &, 650 & \\
\hline descuidado & 3,10 & 1,047 & & ,634 & \\
\hline mentiroso & 3,37 & ,914 & & ,590 & \\
\hline haragán & 3,33 & ,970 & &, 560 & \\
\hline corrupto & 3,29 & 1,080 & &, 543 & \\
\hline inconsciente & 3,24 & ,992 & & ,490 & \\
\hline dejado & 3,32 & 1,006 & & ,479 & \\
\hline conformista & 3,46 & 1,038 & & ,439 & \\
\hline racista & 2,30 & 1,086 & & & ,676 \\
\hline drogas & 2,31 & 1,110 & & & ,636 \\
\hline indiferente & 2,70 & 1,127 & & & ,632 \\
\hline egoísta & 2,79 & 1,111 & & &, 544 \\
\hline fiestero & 2,06 & 1,098 & & &, 344 \\
\hline
\end{tabular}

Se calculó además el puntaje de cada participante con respecto a cada uno de los factores al promediar los reactivos que lo componen. Con base en esa puntuación se calculó la matriz de correlaciones entre factores. Se puede observar una correlación positiva muy baja del factor El europeo siendo responsable con Irresponsabilidad hacia otros de 0,126 . Además hay otra correlación positiva significativa entre la 
Irresponsabilidad Normativa y la Irresponsabilidad hacia otros de 0,253. Hay una correlación negativa significativa entre los factores El europeo siendo responsable y la Irresponsabilidad Normativa (ver tabla 30)

\begin{tabular}{|l|l|}
\hline \multicolumn{3}{|c|}{ TABLA 30 } \\
CORRELACIONES ENTRE FACTORES AUTOCONCEPTO \\
DE RESPONSABILIDAD DEL EUROPEO
\end{tabular}

\section{DISCUSIÓN}

La aplicación de estos instrumentos permite comprender mejor el campo de la representación y hacia dónde están orientados estos datos. De acuerdo al concepto de responsabilidad que Bierhoff y Auhagen (2001) se plantea que se puede observar tres de los elementos que proponen: ser responsable de algo, hacia alguien, y en relación con una instancia. En el grupo SAM de Yo responsable, se pueden ver elementos de ser responsable de algo como: el estudio, el trabajo y las tareas. Ser responsable hacia alguien: siendo honesto, respetuoso y atento. Ser responsable en relación con una instancia podría denominarse Responsabilidad Normativa: siendo puntual, cumplido, ordenado, trabajador, organizado, dedicado, estudioso, comprometido, disciplina. Lo anterior se puede observar en la variabilidad de las puntuaciones individuales que se observaron en los factores Responsabilidad Normativa y Responsabilidad hacia otros en el análisis factorial de Yo responsable (ver tabla 19). Reactivos como ser cumplido, comprometido, trabajador, organizado, dedicado son parte de la dimensión de la Responsabilidad Normativa. Como lo afirma Triandis (1995a) en relación al énfasis a las normas en el estudio del colectivismo, se puede observar que los sujetos escribieron una mayor cantidad de palabras en el factor Responsabilidad Normativa. Otros reactivos como ser limpio, inteligente, respetuoso, atento forman parte de la dimensión de la Responsabilidad hacia otros. Como se puede observar, la multiplicidad de significados no está alejado de su raíz etimológica, tal y como lo señala Winston (2001) sobre el concepto occidental moderno de la responsabilidad en occidente. En los resultados del dendrograma de Yo responsable, se puede apreciar la estructura común a los individuos, identificando dos grandes grupos de palabras, el grupo A conformado por reactivos del orden de la responsabilidad normativa y el grupo B por reactivos de la responsabilidad hacia otros. Sin embargo, dentro del grupo A, existe un subgrupo que está más relacionado con la responsabilidad hacia otros conformado por los reactivos correcto, honesto y confiable (ver tabla 13) Es importante denotar que los estudiantes de humanidades están mayormente orientados hacia lo relacional que los estudiantes de 
ingenierías para esta misma área. En el grupo SAM de Yo responsable le dieron un mayor peso semántico a las palabras definidoras para lo relacional que los estudiantes de ingenierías como respetuoso y atento. Los estudiantes de ingenierías están mayormente orientados hacia lo normativo que a lo relacional, dándole mayor peso semántico a palabras como trabajador, estudioso, disciplina en lo normativo, como se puede ver en la tabla 2. Es probable que lo anterior sea producto del tipo de actividad que desarrollan durante sus estudios universitarios, ya que el área de humanidades está mayormente orientada a trabajar con personas.

Cuando se trata a nivel endogrupal, al evaluar la responsabilidad del guatemalteco, se incorporan nuevas palabras definidoras que no aparecen en Yo responsable, como son amable, patriota y solidario. En el grupo SAM de Guatemalteco responsable, se pueden ver elementos de ser responsable de algo como: el trabajo. Ser responsable hacia alguien: siendo respetuoso, honesto, patriota, solidario, amable y con la familia. La responsabilidad Normativa: siendo trabajador, puntual, cumplido, ordenado, organizado, dedicado (ver tabla 3). Lo anterior también se puede observar en los dos factores Responsabilidad Normativa: esfuerzo, dedicado, trabajador, luchador, ordenado. Como lo afirma Triandis (1995a) en relación al énfasis a las normas en el estudio del colectivismo, se puede observar que los sujetos escribieron una mayor cantidad de palabras en el factor Responsabilidad Normativa, haciendo una inferencia de responsabilidad con mayor énfasis en este factor. Además el factor Responsabilidad hacia otros: siendo amable, inteligente, educado y limpio (ver tabla 20). Dichos factores se obtuvieron en el análisis factorial de la palabra estímulo Guatemalteco responsable. En los resultados del dendrograma se puede observar que también se siguen manteniendo dos grandes grupos, el grupo A relacionado con lo normativo como ser cumplido, dedicado, esfuerzo, trabajador, trabajo, consciente, luchador y otras. El grupo B formado por reactivos del orden de la responsabilidad hacia otros como: amable, inteligente, educado, limpio, respetuoso, solidario, ayudar (ver tabla 14) También se puede apreciar en este dendrograma que la palabra patriota es la que menos relación tiene con el resto de las palabras definidoras. Es importante denotar que tanto los estudiantes de humanidades como de ingenierías consideraron de igual importancia la dimensión de la Responsabilidad Normativa en las palabras trabajador, puntual y cumplido, dándoles un mayor peso semántico. Sin embargo los estudiantes de humanidades les dieron un mayor peso semántico a reactivos de la Responsabilidad hacia otros como honrado, patriota, amable y familia, como se puede ver en la tabla 4.

A nivel exogrupal, al evaluar la responsabilidad del europeo, se incorporan las palabras definidoras: exitoso y cultura que no aparecen en Yo responsable ni en Guatemalteco responsable. En el grupo SAM de Europeo responsable, se pueden ver elementos de ser responsable de algo: el trabajo, la cultura. Ser responsable hacia alguien: siendo respetuoso, educado, honesto, limpio, amable. La responsabilidad normativa como: ser puntual, trabajador, organizado, cumplido, ordenado, estudioso (ver tabla 5). En la responsabilidad normativa en el análisis factorial de la palabra estímulo Europeo responsable, se pueden ver reactivos como: cumplido, puntual, dedicado, trabajador, organizado, comprometido y ordenado (ver tabla 21). Como lo afirma Triandis (1995a) en relación al énfasis a las normas en el estudio del colectivismo, se puede observar que los sujetos escribieron una mayor cantidad de palabras en el factor Responsabilidad Normativa, atribuyendo responsabilidad con mayor énfasis en este factor, así como lo hicieron a nivel personal y endogrupal. En los resultados del análisis de cluster jerárquico con los dendrogramas, se pueden observar 
dos grandes grupos, el normativo con reactivos como dedicado, perseverante, emprendedor, comprometido, cumplido, ordenado y otras. Y la dimensión de la responsabilidad hacia otros, como amable, limpio y patriota. También puede considerarse que el grupo A tiende a una división, en donde el primer grupo A1 manifiesta elementos puramente normativos, como dedicado, perseverante, exitoso y otros. Y el grupo A2 manifiesta elementos de responsabilidad hacia otros se pueden ver reactivos como educado, honesto, justo, respetuoso (ver tabla 15).Es importante denotar que los estudiantes de ingenierías le dieron un mayor peso semántico a palabras de la dimensión de la responsabilidad hacia otros como: respetuoso, honesto y limpio que los estudiantes de humanidades. En este caso, los estudiantes de humanidades le dieron mayor peso semántico a palabras de la dimensión de la responsabilidad normativa como: organizado, ordenado y estudioso que los estudiantes de ingenierías como se puede ver en la tabla 6 . Es probable que lo anterior sea producto de la percepción que los estudiantes de ingeniería tienen sobre las actividades de las empresas europeas en el país y posiblemente también por experiencia directa con europeos en Guatemala o en el extranjero, ya que los estudiantes de ingenierías conocen a más europeos.

Al evaluar Yo irresponsable, se encontró que hubo una mayor riqueza semántica, así como para Guatemalteco irresponsable, que fueron las dos palabras estímulo que obtuvieron los mayores valores J. En el grupo SAM para Yo irresponsable se pueden ver elementos de ser irresponsable hacia alguien: siendo irrespetuoso, deshonesto, mentiroso y malo. Así como elementos de la irresponsabilidad normativa: ser impuntual, desordenado, haragán, desorganizado, incumplido, perezoso, dejado, olvidadizo (ver tabla 7). En el análisis factorial puede observarse que las dos dimensiones Irresponsabilidad Normativa contiene reactivos como: haragán, conformista, dejado, despreocupado, indiferente, incumplido, fracaso, desinteresado y olvidadizo. Como lo afirma Triandis (1995a) en relación al énfasis a las normas en el estudio del colectivismo, se puede observar que los sujetos escribieron una mayor cantidad de palabras en el factor Irresponsabilidad Normativa. Y la dimensión de irresponsabilidad hacia otros se encuentran reactivos como: malo, sucio, egoísta, deshonesto e irrespetuoso (ver tabla 22). En los resultados del dendrograma se pueden observar 2 grandes grupos, la irresponsabilidad normativa y la irresponsabilidad hacia otros, conformado este último por reactivos como incapaz, negativo, egoísta, malo, sucio, deshonesto, irrespetuoso y mentiroso. (ver tabla 16) Tanto los estudiantes de humanidades como los de ingeniería, les dieron mayor peso semántico a más palabras de la irresponsabilidad normativa que a la irresponsabilidad hacia otros, como se puede ver en la tabla 8 .

Al nivel endogrupal, al evaluar la irresponsabilidad del Guatemalteco, se incorporan las palabras definidoras: ladrón, corrupto y vago que no aparecen en Yo irresponsable. En el grupo SAM de Guatemalteco irresponsable, se pueden ver elementos que corresponden a ser irresponsable hacia alguien como: siendo irrespetuoso, mentiroso, deshonesto, ladrón, corrupto. Ser irresponsable de acuerdo a lo normativo comprende reactivos como: ser impuntual, haragán, desordenado, incumplido, perezoso, desorganizado, desinteresado, dejado, despreocupado (ver tabla 9). En el análisis factorial de Guatemalteco irresponsable se pueden observar dos dimensiones Irresponsabilidad hacia otros con elementos como: maleducado, irrespetuoso, ser egoísta, violencia, mentiroso, corrupto, deshonesto, ignorante y ladrón. A nivel normativo hay reactivos como: ser dejado, perezoso, despreocupado, haragán, conformista, desorganizado, desordenado, impuntual (ver tabla 23). Como lo afirma 
Triandis (1995a) en relación al énfasis a las normas en el estudio del colectivismo, se puede observar que los sujetos escribieron una mayor cantidad de palabras en el factor Irresponsabilidad hacia otros, sin embargo la media del factor Irresponsabilidad Normativa fue más significativo que el factor Irresponsabilidad hacia otros que tuvo una posición ligeramente inferior a la media. Según los resultados del análisis de cluster jerárquico, hay un mayor consenso por la Irresponsabilidad hacia otros en reactivos como irrespetuoso, maleducado, deshonesto, inconsciente, sucio, borracho, ladrón, egoísta, violencia, corrupto y mentiroso. Sin embargo desempleado e ignorante son las palabras más alejadas del resto de lo relacional (ver tabla 17). Es importante denotar que los estudiantes de ingenierías le dieron un mayor peso semántico a más palabras del orden de la irresponsabilidad hacia otros como mentiroso, corrupto y ladrón que los estudiantes de humanidades como se puede ver en la tabla 10.

A nivel exogrupal, al evaluar la irresponsabilidad del europeo, se puede ver que se incorporan palabras como: drogas y fiestero, que no aparecen en Yo irresponsable ni en Guatemalteco irresponsable. En el grupo SAM de Europeo Irresponsable se pueden ver elementos de ser irresponsable con algo como: las drogas. Ser irresponsable hacia alguien como: ser irrespetuoso, ser sucio, fiestero y egoísta. Hay elementos de la irresponsabilidad normativa como: ser impuntual, haragán, desordenado, desorganizado, incumplido, desinteresado, dejado, descuidado y perezoso (ver tabla 11). En el análisis factorial de la palabra estímulo Yo irresponsable, se encontraron dos factores Irresponsabilidad normativa con reactivos como: dejado, incumplido, perezoso, despreocupado, desorganizado, descuidado, haragán, impuntual, desinteresado, vago, conformista y desordenado. Como lo afirma Triandis (1995a) en relación al énfasis a las normas en el estudio del colectivismo, se puede observar que los sujetos escribieron una mayor cantidad de palabras en el factor Irresponsabilidad Normativa. Elementos de la irresponsabilidad hacia otros como ser racista y egoísta forman parte de esta dimensión (ver tabla 24). A través del dendrograma, se puede apreciar con mayor claridad la división de lo relacional con lo normativo con respecto a la Irresponsabilidad del europeo. Parte de lo relacional como ser ignorante, sucio, egoísta, racista y fiestero son parte de la categoría de la Irresponsabilidad hacia otros, sin embargo hay un subgrupo A, que también cuenta con elementos relacionales, en el grupo A2, dentro de reactivos de carácter normativo (ver tabla 18) Si se comparan los estudiantes de ingenierías con los de humanidades, se puede apreciar que los estudiantes de ingenierías le dan mayor peso semántico a más palabras definidoras de la dimensión irresponsabilidad hacia otros que los estudiantes de humanidades como se puede ver en la tabla 12.

De acuerdo al análisis factorial exploratorio del autoconcepto de responsabilidad, los estudiantes se consideraron altamente responsable, es decir correctos, inteligentes, trabajadores, atentos, confiables y conscientes. (ver tabla 25) Se consideraron responsables hacia la familia. Además se consideraron casi nada irresponsables hacia otros, es decir casi nada malos, sucios, deshonestos, egoístas, mentirosos, incapaces o negativos. Como lo afirma Triandis (1995a) en relación al énfasis a las metas grupales en el estudio del colectivismo, se puede observar que los sujetos escribieron una mayor cantidad de palabras de índole relacional en el factor Yo siendo responsable. Tanto la dimensión anterior como Irresponsabilidad hacia otros fueron significativas, lo que sugiere que lo mencionado por Hofstede (2001) que la población guatemalteca es altamente orientada a lo colectivista y con sólidas relaciones, ya que los sujetos se perciben a sí mismos como altamente responsables y nada irresponsables hacia otros. 
A nivel endogrupal, a partir del análisis factorial exploratorio del autoconcepto de responsabilidad del guatemalteco, los estudiantes consideraron al guatemalteco algo responsable hacia otros, a través de ayudar, siendo amables, solidarios y con la familia. Los demás factores fueron neutros (ver tabla 27). Sin embargo de acuerdo al análisis de medias los sujetos consideraron al guatemalteco altamente orientado a la familia, inteligente y amable, así como violento e impuntual. Como lo afirma Triandis (1995a) en relación al énfasis a las metas grupales en el estudio del colectivismo, se puede observar que los sujetos escribieron una mayor cantidad de palabras de índole relacional.

A nivel exogrupal, los sujetos consideraron al europeo como responsable, tanto a nivel normativo como relacional, considerando reactivos como ser dedicado, perseverante, estudioso, inteligente, organizado, comprometido, ordenado, exitoso, educado, honesto, emprendedor, trabajo, cumplido, culto, cultura, consciente, justo y limpio (ver tabla 29). De acuerdo al análisis de medias los sujetos consideraron al europeo altamente educado, es decir con instrucción formal, puntual, orientado a la cultura y estudioso. Como lo afirma Triandis (1995a) en relación al énfasis a las normas en el estudio del colectivismo, se puede observar que los sujetos escribieron una mayor cantidad de palabras de índole normativo en el factor El europeo siendo responsable.

Cabe mencionar que los estudiantes se describieron mejor en la responsabilidad de rol de estudiante, ya que utilizaron palabras definidoras más acorde a asumir el papel determinado. Es posible que esto haya influido en una mayor cantidad de palabras definidoras de índole normativo en casi todas las palabras estímulo, ya que según Hart (1968) menciona que la responsabilidad de rol se extiende a incluir tareas asignadas a una persona por muto acuerdo o de algún otro modo. Es probable que sea también por el énfasis a las normas, según Triandis (1995a) en su propuesta del colectivismo. También es posible que los sujetos hayan inferido responsabilidad en cada caso en función de factores disposicionales, tal y como lo menciona Watson (1982) en relación al sesgo fundamental de atribución. Lo anterior quiere decir que los sujetos se hayan atribuido responsables a sí mismos, al guatemalteco y al europeo en función de ciertos rasgos individuales que deberían tener.

A partir de lo anterior se comprueba la multiplicidad de significados del concepto de responsabilidad en la dinámica Ego-Alter-Objeto.

Es interesante observar que el $12,4 \%$ de los padres de los sujetos era gerente, así como el 7,3\% era empresario, sumando un 19,7\%. Lo anterior denota el horizonte de oportunidades para los sujetos en el mundo empresarial, ya que es probable que los sujetos opten por puestos similares en el futuro, lo que permite predecir un modelo de responsabilidad con el que han sido educados. 


\section{BIBLIOGRAFÍA}

Bartos, Frantisek Individualism and Collectivism of Czech Population and their Relation to Narcissism. Slovak Sociological Review. Vol. 42 (No. 2: 134-161)

Bierhoff, H.W. \& Auhagen, A.E. (2001). Responsibility as a fundamental human phenomenon. In A.E. Auhagen \& H.W. Bierhoff (Eds.), Responsibility. The many faces of a social phenomenon (pp. 1-8). London: Routledge.

Bolasco, S. (1999). Analisi multidimensionale dei dati: metodi, strategie e criteri d'interpretazione. Roma: Carocci

Brushlinski A (1994) Problems of the psychology of the subject. Rossijskaja Akademia Nauk, Moscow

Fauconnet, P. (1920). La Responsabilité. Parigi: Alcan.

Fincham, F.D. \& Jaspars, J.M. (1980). Attribution of responsibility: from man the scientist to man as lawyer. In L. Berkowitz (Ed.), Advances in experimental social psychology (vol. 13, pp. 81-138). San Diego, CA: Academic press.

Hamilton, V.L. (1978). Who is responsible? Toward a social psychology of responsibility attribution. Social Psychology, 41, 316-328.

Hamilton, V.L. (1986). Chains of command: responsibility attribution in hierarchies. Journal of Applied Social Psychology, 16, 118-138.

Hart, H.L.A. (1968). Punishment and Responsibility. Oxford: Oxford University Press.

Heider, F. (1958). The psychology of interpersonal relations. New York: Wiley.

Hewstone, M. (1989). Causal attribution. Oxford: Bleckwell. 
Hofstede, G. (2001). Culture's consequences: Comparing values, behaviors, institutions and organizations across nations (2nd ed.). Beverly Hills, CA: Sage.

Jones, E.E. \& Davis, K.E. (1965). From Acts to Dispositions: The Attribution Process in Person Perception. In L. Berkowitz (ed.), Advances in Experimental Social Psychology (Vol. 2, pp. 219-266). New York: Academic Press.

Kelley, H.H. (1967). Attribution theory in social psychology. In D. Levine (Ed.), Nebraska symposium on motivation (vol. 15, pp. 192-238). Lincoln: University of Nebraska press.

Lebart, L. \& Salem, A. (1988). Analyse statistique des donnees textuelles: questions ouvertes et lexicometrie. Paris: Dunod.

Markova, I. (2003). Dialogicality and social representations. Cambridge: Cambridge University Press.

Markus, H., and Kitayama, S. (1991) Culture and the self: Implications for cognition, emotion and motivation, Psychological Review 98: 224-254

McKeon, R. (1957). The development and the Significance of the Concept of Responsibility. Revue Internationale de Philosophie, 39, 3-32.

Moscovici, S. (1981). On social representations. In J.P. Forgas (Ed.), Social cognition, perspectives on everyday understanding (pp. 181-210). London: Academic Press.

Moscovici, S. (1984). Psychologie Sociale. Paris: PUF.

Nisbett, R.E. (1998) Essence and accident, in J.M. Darley and J.Cooper (eds), Attribution in Social Interaction: The legacy of Ned Jones, pp.171-197, Washington, DC: American Psychological Association.

Scott, M.B. \& Lyman, S. (1968). Accounts. American Sociological Review, 33, 46-62.

Schlenker, B.R., Britt, T.W., Pennington, J., Murphy, R. \& Doherty, K. (1994). The triangle model of responsibility. Psychological Review, 101, 101-113. 
Schulz, W. (1988). Le nuove vie della filosofia contemporanea, vol. 5, Responsabilità. Genova: Marietti.

Shweder, R.A., and Bourne, E.J. (1982) Does the concept of the person vary crossculturally? In A.J.Marsella and G.M.White (eds), Cultural Conceptions of Mental Health and Therapy, Dordrech, Holland: D.Riedel

Triandis, H. (1989) The self and social behavior in different cultural contexts, Psychological Review 96:506-520

Triandis, H. C. (1995a). Individualism and collectivism. Boulder, CO: Westview Press.

Triandis, H. C. (1995b). Motivation and achievement in collectivist and individualist cultures. In M. L. Maehr \& P. R. Pintrich (Eds.), Advances in motivation and achievement (pp. 1-30). Greenwich, CT: JAI Press.

Villegas-Reimers, "Judgments of Responsibility: Their Relationship with Self and Moral Reasoning in Venezuelan Adolescents,” DAE 49 (1989): 2597A (Harvard Graduate School of Education, 1988).

Watson, J.B. (1924). Behaviorism. Nueva York: Norton

Winston, Davis. (2001). Taking responsibility. Comparative Perspectives. The University Press of Virginia. 5-10

Zamperini, A. (1998). Psicologia Sociale della Responsabilità. Torino: UTET. 
Revista Internacional de Psicología www. revistapsicologia.org

ISSN 1818-1023
Vol. 11 No. 2

Instituto de la Familia Guatemala

Julio 2010

\section{(1) (1)}

Este texto está protegido por una licencia CreativeCommons

4.0.

Usted es libre para Compartir - copiar y redistribuir el material en cualquier medio o formato - y Adaptar el documen- to -remezclar, transformar y crear a partir del material- para cualquier propósito, incluso comercialmente, siempre que cumpla la condición de:

Atribución: Usted debe reconocer el crédito de una obra de manera adecuada, proporcionar un enlace a la licencia, e in- dicar si se han realizado cambios. Puede hacerlo en cualquier forma razonable, pero no de forma tal que sugiera que tie- ne el apoyo del licenciante o lo recibe por el uso que hace.

$\underline{\text { Resumendelicencia - Textocompletodelalicencia }}$ 